ARTICLE

\title{
Antibacterial effects of nanopillar surfaces are mediated by cell impedance, penetration and induction of oxidative stress
}

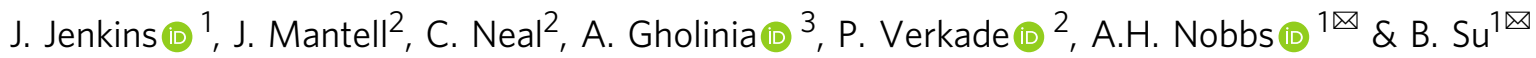

\begin{abstract}
Some insects, such as dragonflies, have evolved nanoprotrusions on their wings that rupture bacteria on contact. This has inspired the design of antibacterial implant surfaces with insectwing mimetic nanopillars made of synthetic materials. Here, we characterise the physiological and morphological effects of mimetic titanium nanopillars on bacteria. The nanopillars induce deformation and penetration of the Gram-positive and Gram-negative bacterial cell envelope, but do not rupture or lyse bacteria. They can also inhibit bacterial cell division, and trigger production of reactive oxygen species and increased abundance of oxidative stress proteins. Our results indicate that nanopillars' antibacterial activities may be mediated by oxidative stress, and do not necessarily require bacterial lysis.
\end{abstract}

\footnotetext{
${ }^{1}$ Bristol Dental School, University of Bristol, Bristol, UK. ${ }^{2}$ School of Biochemistry, University of Bristol, Bristol, UK. ${ }^{3}$ School of Materials Science, University of Manchester, Manchester, UK. ${ }^{凶}$ email: angela.nobbs@bristol.ac.uk; b.su@bristol.ac.uk
} 
$t$ is now well established that insect wings, including the cicada and dragonfly, possess antibacterial and antifungal properties. Previous studies indicate that this process is mediated by the physical nanoprotrusions found on the wing surface, which ultimately stretch and damage the microbial cell upon contact, leading to lysis and death ${ }^{1-6}$. This effect was first observed in Pseudomonas aeruginosa, where individual bacteria were shown to sink and spread between the nanopillars found on Psaltoda claripennis (cicada) wings, occurring in attachment/killing cycles of $20 \mathrm{~min}$ (ref. ${ }^{1}$ ); this mechanism was proposed to occur by mechanical rupture of the cell. Bactericidal effects were also described for other Gram-negative bacteria, including Branhamella catarrhalis, Escherichia coli and Pseudomonas fluorescens ${ }^{6}$. Alongside cicada, dragonfly wings have been shown to possess efficient bactericidal properties. Diplacodes bipunctata wings were found to mediate killing of both Gram-negative ( $P$. aeruginosa) and Gram-positive (Staphylococcus aureus and Bacillus subtilis) bacteria. The capillary architecture of $D$. bipunctata wing nanoprotrusions is hypothesised to enhance cell wall stress and deformation, thereby extending bactericidal activity towards Gram-positive cell types ${ }^{2}$.

The unique bactericidal properties of cicada and dragonfly wings have drawn significant research interest ${ }^{7-10}$, as the physical nature of bacterial killing could provide an effective strategy to prevent biofilm formation, and infection of indwelling and implantable devices, while negating the current need to use materials impregnated with antibiotics. To date, a wide range of nanofabrication techniques have been utilised to generate bactericidal nanotopographies on synthetic materials, including black silicon $(\mathrm{bSi})^{2}$, titanium ${ }^{11}$, titanium alloy ${ }^{12}$ and polymers ${ }^{13}$.

Several models for the process of contact killing have been proposed. The biophysical model suggested that cicada wing nanopillars induce physical stretching of the cell membrane upon contact, leading to bacterial rupture and death, and cell rigidity was reported to be an important determinant of susceptibility ${ }^{14}$. In support of this, the elastic mechanical model proposed that Gram-positive bacteria are less susceptible to nanopillar deformation and rupture owing to their lower maximum stretching capacity. However, by increasing nanopillar sharpness and spacing, the antibacterial properties may be enhanced ${ }^{15}$. A quantitative thermodynamic model proposed that the bactericidal activity of nanopatterned surfaces is directly related to the balance between adhesion energy and deformation energy. The model predicts that within certain dimensions (nanopillar radius $0-50$ $\mathrm{nm}$ and nanopillar spacing $100-250 \mathrm{~nm}$ ), the stretching degree applied across the bacterial envelope is enhanced by nanoarrays with greater nanopillar diameters $(50 \mathrm{~nm})$ and reduced nanopillar spacing $(100 \mathrm{~nm})^{16}$.

Multiple factors are reported to influence the antimicrobial efficacy of natural and synthetic nanopillars. Of note, the microbial adhesion force to a nanotopography has been shown to directly influence viability. Saccharomyces cerevisiae rupturing was greatest in strains that adhered most strongly to cicada wing nanopillars ${ }^{5}$. Similarly, the strong adhesion between dragonfly nanopillars and E. coli extracellular polymeric substance is reported to promote bacterial membrane damage ${ }^{4}$. The rigidity of bacterial cells has also been found to influence their susceptibility to mechanical rupture ${ }^{6,14}$, whereby Gram-negative bacteria were more sensitive to nanopillar-mediated stretching. This observation most likely reflects variations in envelope architecture. Gram-negative envelopes consist of an outer and inner membrane, separated by a thin $(\approx 5 \mathrm{~nm})$ peptidoglycan cell wall that occupies the periplasmic space. By contrast, the Gram-positive bacterial cell wall is significantly thicker $(\approx 20-100 \mathrm{~nm})^{13,17}$, which may reduce stretching sensitivity. In addition to this, nanotopography geometries, including aspect ratio and nanopillar density, have been shown to influence the efficiency of bactericidal activity $3,5,13,18$.

Although the bactericidal activity of natural and synthetic nanopillars has been widely reported, no consensus has been reached on the precise mechanism that leads to the microbial cell death and importantly, while many studies infer that nanopillars mediate mechanical rupture of bacterial cells, this has not been shown conclusively. In addition to their capacity to rupture and lyse bacteria, nanostructured surfaces are reported to alter the genomic and proteomic profile of bacteria. Of note, type-1 fimbriae expression was significantly downregulated in $E$. coli adhered to gold nanoparticle surfaces, while stress response proteins involved in protection from DNA and membrane damage had been upregulated 19,20 . Consistent with these findings, E. coli incubated on single-walled carbon nanotubes (CNTs) expressed high levels of stress response proteins related to cell membrane damage and oxidative stress ${ }^{21}$. Physiological responses of this nature may contribute significantly to the overall bactericidal activity of nanostructured surfaces. Elucidating these molecular changes is therefore essential to progress our understanding of the mechanistic processes that lead to bacterial cell death.

To better understand and validate the antimicrobial mechanisms relating to specific nanotopographies, this study set out to determine the morphological and physiological responses of bacteria to dragonfly mimetic nanopillars, as these nanotopographies have been reported to possess highly efficient bactericidal activity against both Gram-negative and Gram-positive bacteria $^{2,4}$. To elucidate the impact of titanium dioxide $\left(\mathrm{TiO}_{2}\right)$ nanopillars on bacterial envelope integrity, and to determine whether nanopillars induce mechanical rupture and cell lysis, electron tomography techniques were utilised to reconstruct detailed 3D visualisations of bacteria adhered to nanopillars. To gain a deeper insight into the molecular changes that occur on nanopillar surfaces, changes in protein expression were investigated by quantitative tandem mass tagging (TMT) proteomic analysis. Bacterial cell viability (BacTiter-Glo, RealTime-Glo) assays were additionally employed to quantify the growth and viability of $S$. aureus, E. coli and Klebsiella pneumoniae populations incubated with $\mathrm{TiO}_{2}$ nanopillars.

\section{Results}

Characterisation of $\mathrm{TiO}_{2}$ nanopillars on titanium substrate. A thermal oxidation technique $\mathrm{e}^{12,22}$ was used to generate nanopillars on grade 5 titanium alloy (Ti-6Al-4V), a material widely used for orthopaedic implants ${ }^{23}$. Nanopillar surface NW-850-5 was oxidised at $850{ }^{\circ} \mathrm{C}$ for $5 \mathrm{~min}$ (Fig. 1), generating a nanotopography closely mimicking the nanoprotrusions found on dragonfly wings. Nanopillars consisted of $\mathrm{TiO}_{2}$ and were predominantly comprised of rutile $\mathrm{TiO}_{2}$ (98\%; Supplementary Figs. 1-2).

$\mathrm{TiO}_{2}$ nanopillars induce envelope deformation and penetration. The bactericidal action of cicada wing nanopillars was reported to occur immediately after seeding with $P$. aeruginos $a^{1}$. Furthermore, the killing rates observed on dragonfly wing mimetic bSi were highest within $3 \mathrm{~h}$ (ref. ${ }^{2}$ ). These conclusions were partly drawn from qualitative analysis, using scanning electron microscopy (SEM), which revealed significant changes in bacterial morphology. To enable a direct comparison with these studies, high-resolution SEM was used to determine whether $\mathrm{TiO}_{2}$ nanopillars induced mechanical rupture and lysis of $S$. aureus, E. coli or K. pneumoniae after $3 \mathrm{~h}$.

On control surfaces, bacteria exhibited the characteristic morphologies of coccoid (S. aureus) or bacillus (E. coli and $K$. pneumoniae) cells after 3 -h incubations (Fig. 2). These cell 

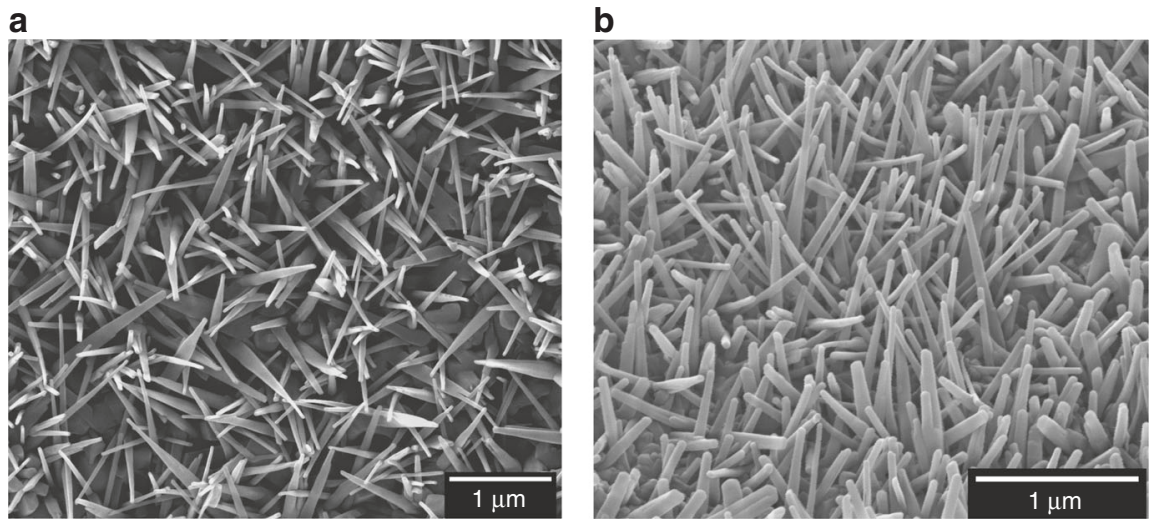

Fig. 1 Characterisation of $\mathrm{TiO}_{\mathbf{2}}$ nanopillars. Scanning electron micrographs of $\mathrm{TiO}_{2}$ nanopillar surface NW-850-5 from a top view a and $40^{\circ}$ stage tilt $\mathbf{b}$. $\mathrm{NW}-850-5$ was generated at $850^{\circ} \mathrm{C}$ for a duration of $5 \mathrm{~min}$. Scale bars: $1 \mu \mathrm{m}$. Micrographs are representative of three thermal oxidation batches $(n=3)$, each batch containing 25 surfaces.
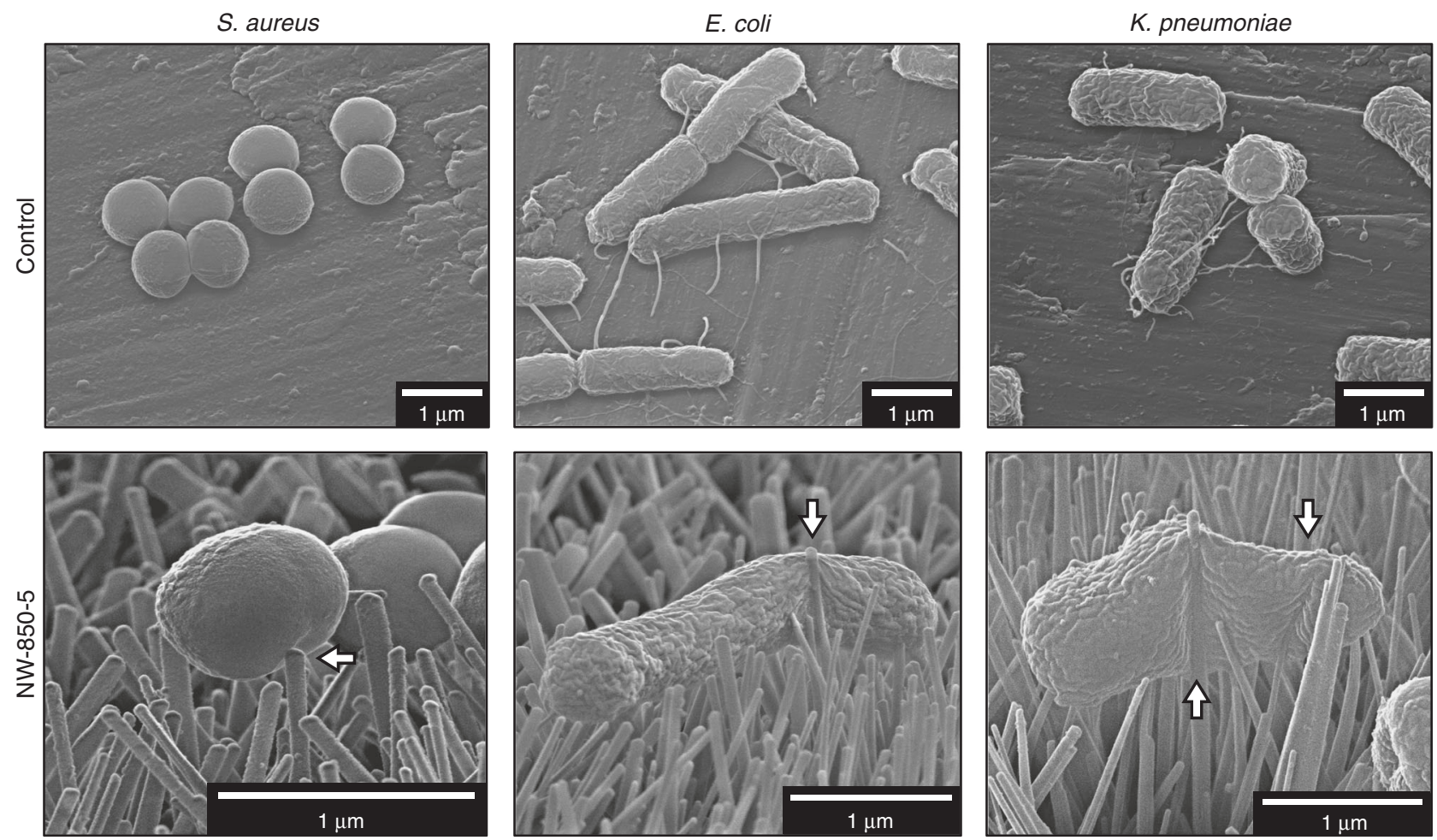

Fig. 2 Determining bacterial morphology on nanopillar surface NW-850-5 using SEM. Scanning electron micrographs of Gram-positive (S. aureus) or Gram-negative (E. coli and K. pneumoniae) bacteria following 3-h static incubation on flat titanium alloy (control) and $\mathrm{TiO}_{2}$ nanopillar surface NW-850-5. White arrows highlight regions of nanopillar-induced envelope deformation. Micrographs are representative of three surfaces ( $n=3$ ), with each surface visualised in at least three different areas.

morphologies were also observed after 0.5-, 1- and 10-h incubations (Supplementary Figs. 3-5). The coverage of each bacterium increased on the control surfaces between 3 and $10 \mathrm{~h}$, and there was extensive evidence of microcolony formation, indicative of early stage biofilm formation. In contrast, coverage and density were visibly lower for all bacteria on nanopillar surfaces (Supplementary Figs. 6-8). Overall, the morphologies of S. aureus incubated on NW-850-5 were comparable to bacteria on the controls but envelope deformation was observed, defined here as the process by which nanopillars indent or change the surface morphology of the bacterial envelope in a contactdependent manner. Nanopillar-induced envelope deformation was also observed for E. coli and K. pneumoniae incubated on NW-850-5 (Fig. 2). Despite this, there was no evidence that nanopillars had induced mechanical rupture or lysis of bacterial cells, defined here as the loss of cytosolic content and turgor pressure, resulting in bacteria sinking into the nanotopography. Additionally, the morphologies of bacteria after 0.5-, 1- and 10-h incubations on nanopillar surfaces were comparable to those of bacteria on control surfaces, with only subtle indications of nanopillar-induced envelope deformation for $E$. coli and $K$. pneumoniae. It was also noted that E. coli and $K$. pneumoniae microcolonies appeared to express fimbria-like surface appendages on control surfaces, while these structures were largely absent from bacteria in contact with the nanopillars.

The observation of nanopillar-induced envelope deformation for S. aureus, E. coli and K. pneumoniae was partly consistent with the proposed mechanism of nanopillar-mediated contact 


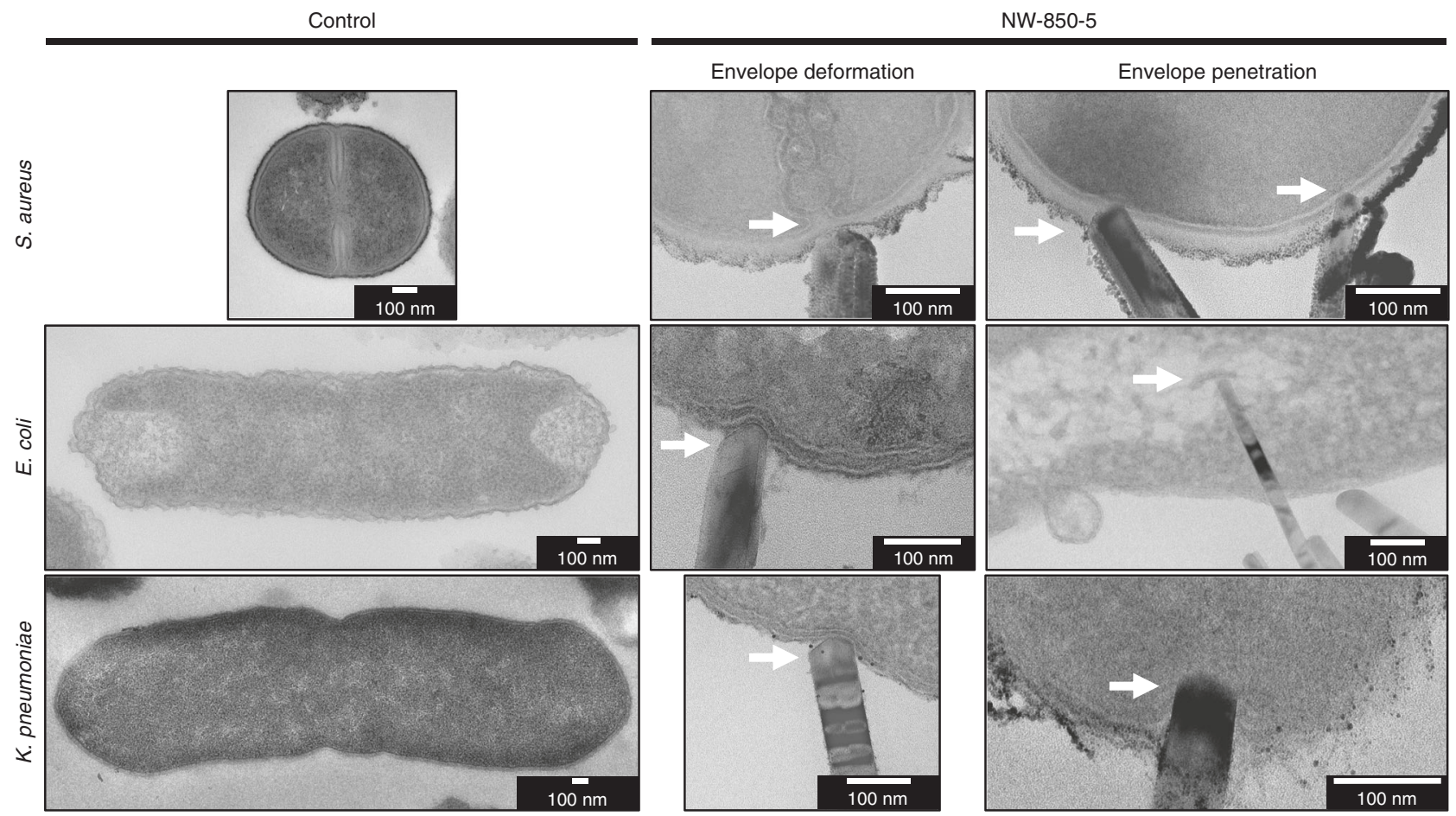

Fig. 3 Nanopillar-induced envelope deformation and penetration in Gram-negative and Gram-positive bacteria. TEM micrographs of S. aureus, E. coli and K. pneumoniae following incubation on control surfaces and NW-850-5 for $3 \mathrm{~h}$. Regions where nanopillars had deformed or penetrated the bacterial envelope have been marked by white arrows. Cross section thickness: $70 \mathrm{~nm}$. Micrographs are representative of three independent surfaces $(n=3)$.

killing, which suggests that as bacteria adhere to the nanopillar surface, the envelope is stretched to the point of physical rupture, leading to cell death ${ }^{14}$. However, it was unclear whether nanopillar-induced deformation could lead to the penetration of the bacterial envelope, defined as the capacity of nanopillars to pierce through the bacterial envelope, thereby disrupting the continuous barrier between the cytosol and extracellular environment. To investigate this in greater detail and determine whether penetration could induce cell lysis, electron microscopy techniques were utilised that could resolve the interface between nanopillars and the bacterial envelope, namely transmission electron microscopy (TEM) and focused ion beam SEM (FIBSEM).

Following recovery from control surfaces, S. aureus displayed a uniform and continuous envelope and appeared to be dividing, as was evident from the bacterial septum (Fig. 3; top left). The characteristic architecture of a Gram-positive envelope was observed, with a plasma membrane (average: 8 $\mathrm{nm} \pm 1 \mathrm{~nm}$ ) surrounded by a peptidoglycan cell wall (average: $22 \mathrm{~nm} \pm 2 \mathrm{~nm}$ ). The envelopes of E. coli and K. pneumoniae were comparable, both consisting of outer $(12 \mathrm{~nm} \pm 2 \mathrm{~nm}$ and $9 \mathrm{~nm} \pm 2 \mathrm{~nm}$, respectively) and inner $(9 \mathrm{~nm} \pm 2 \mathrm{~nm}$ and $6 \mathrm{~nm} \pm$ $1 \mathrm{~nm}$, respectively) membranes, separated by a thin $(\approx 5 \mathrm{~nm})$ peptidoglycan layer (Fig. 3; middle and bottom left). S. aureus ultrastructure was largely unaffected by the interactions with NW-850-5, with envelope deformation and penetration observed at a low frequency (5\%; Fig. 3; top middle and top right). In contrast, TEM analysis provided strong evidence that nanopillars had deformed the envelope of E. coli (Fig. 3; middle and middle bottom); indentations in close proximity to the nanopillar tips were observed in $26 \%$ of cells, while envelope penetration was observed at a frequency of $19 \%$. For $K$. pneumoniae, nanopillars had induced envelope deformation in $11 \%$ of cells and had penetrated $8 \%$ of cells analysed.
To determine whether the envelope deformation was localised to the point of nanopillar contact, or whether global perturbations had occurred, tomography analysis was utilised to monitor changes in envelope structure and shape with distance across the cell. Tomography was performed on an E. coli cross section, measuring $250 \mathrm{~nm}$ in thickness. Initial TEM analysis had identified a single nanopillar interacting with the cell (Fig. 4a). Tomographic slices were extracted from the start, middle and point of nanopillar contact; these highlighted that the envelope structure was uniform at the beginning (Fig. $4 \mathrm{~b}$ ) and middle (Fig. 4c) of the tomogram, while deformation was only seen at point of nanopillar contact (Fig. 4d). This confirmed the localised nature of these interactions. Analysis of the 3D reconstruction of E. coli revealed that the bacterial envelope had been deformed by $\sim 80-100 \mathrm{~nm}$, but this had not resulted in mechanical rupture or lysis (Fig. 4e). Tomography was also performed on a single $K$. pneumoniae cell that appeared to be penetrated by multiple nanopillars (Fig. 5a). Analysis of multiple tomographic slices confirmed that five out of six nanopillars had penetrated, with depths varying from $103-179 \mathrm{~nm}$ (Fig. 5b).

To investigate the effects of nanopillars on bacterial envelope morphology further, cross sectional analysis of E. coli and $S$. aureus was performed using FIB-SEM. FIB-SEM is an effective tool for investigating cell-material interfaces with the ability to selectively analyse individual bacterial cells ${ }^{24,25}$. Preliminary SEM analysis revealed a single nanopillar (nanopillar 1) causing significant envelope deformation on one side of $E$. coli, leading to the bacterium being forced against a nanopillar (nanopillar 2) on the opposite side (Fig. 6). Automated ion beam milling was performed to reveal whether either nanopillar had penetrated the envelope. Although nanopillar 1 had deformed the bacterial envelope by $191 \mathrm{~nm}$, it had not penetrated the cell. Nonetheless, it was evident from such analyses that nanopillars had capacity to act as physical barriers, entrapping bacterial cells and possibly impeding division on the horizontal plane. 
a

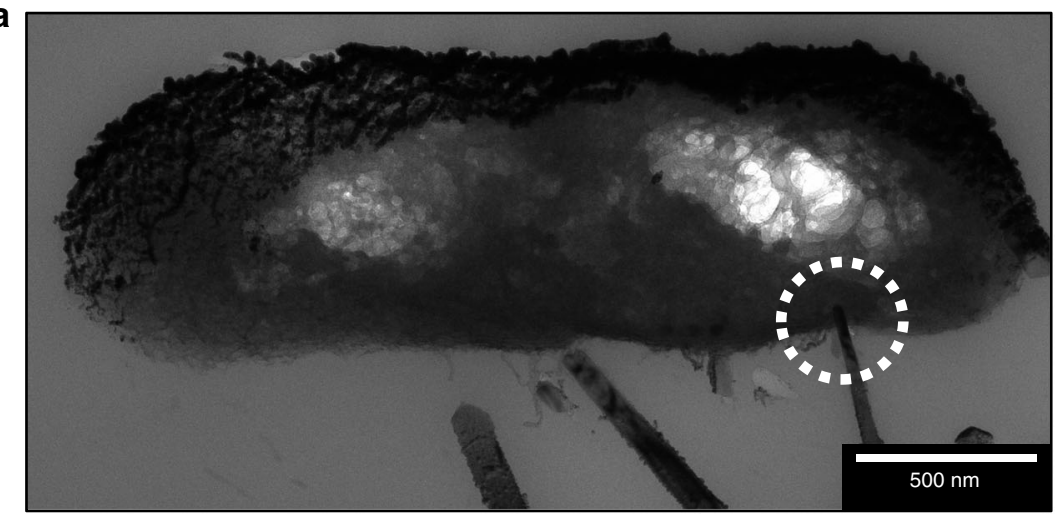

e

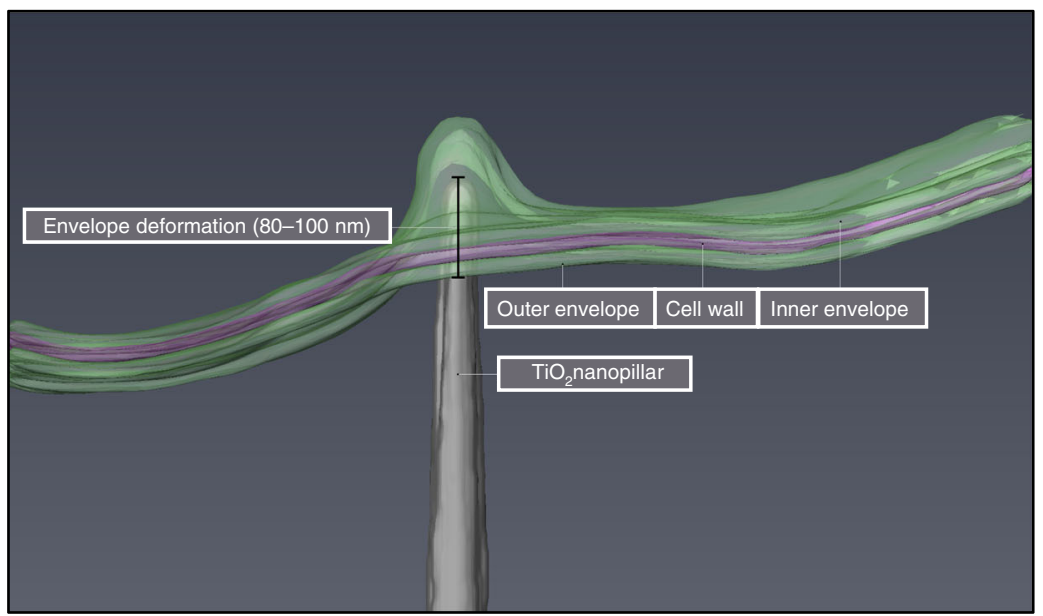

b

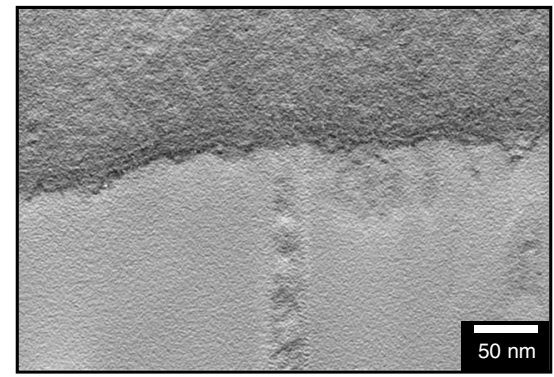

c

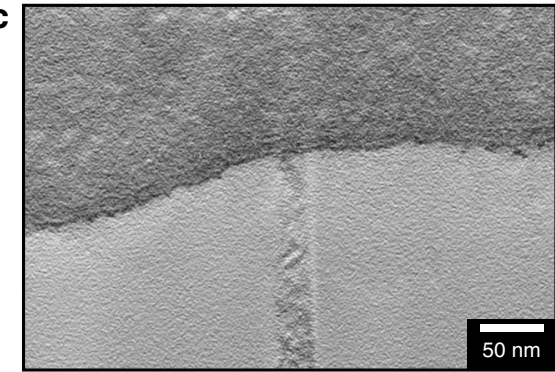

d

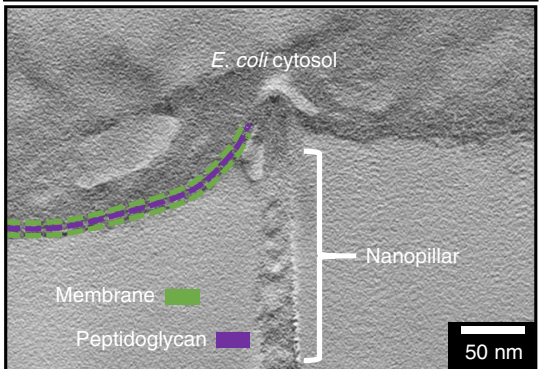

Fig. 4 Tomogram reconstruction of nanopillar-induced envelope deformation in $\boldsymbol{E}$. coli. A tilt series was acquired of $E$. coli $\mathbf{a}$, where a single nanopillar appeared to be penetrating the envelope (dashed white circle). Analysis of tomographic slices from the start $\mathbf{b}$, middle $\mathbf{c}$ and point of nanopillar contact d revealed that the nanopillar had clearly indented the envelope but had not pierced through. The interaction was therefore categorised as nanopillarinduced envelope deformation, localised at the point of nanopillar contact. This interaction is highlighted in the 3D reconstruction shown in e. Electron tomography was performed on three $E$. coli cells interacting with $\mathrm{TiO}_{2}$ nanopillars, these were acquired in two tilt series.

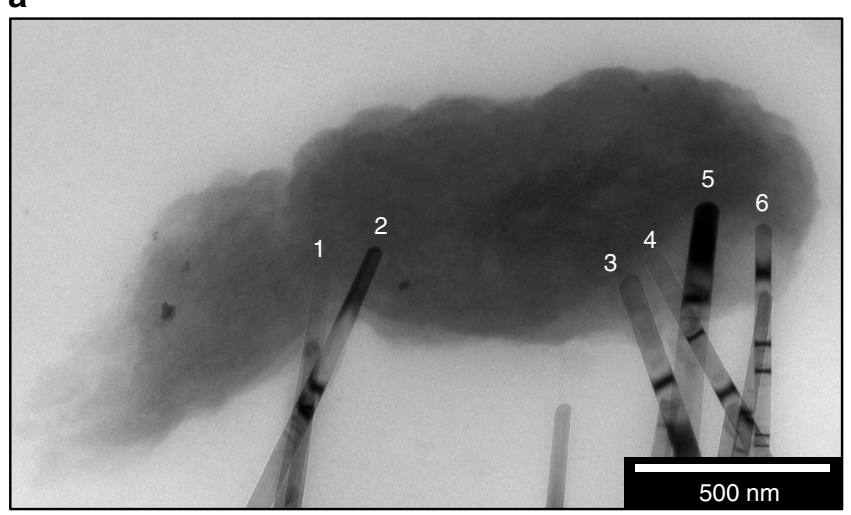

b

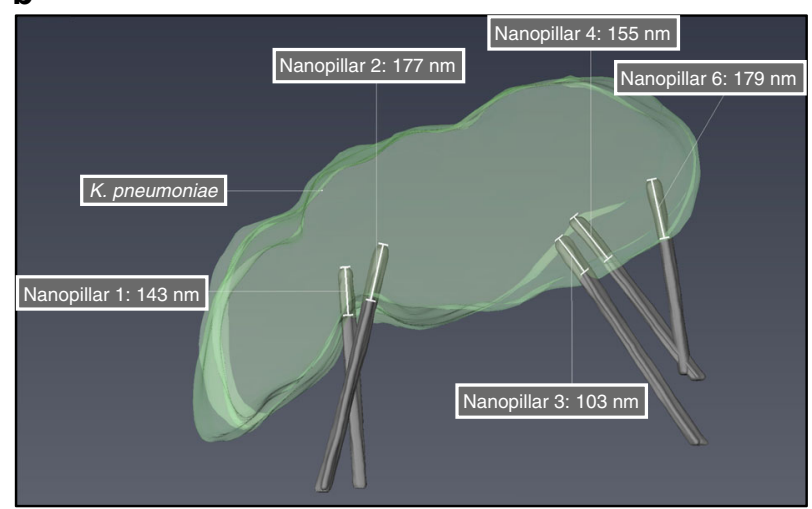

Fig. 5 Tomogram reconstruction of nanopillar-induced envelope penetration in $\boldsymbol{K}$. pneumoniae. A tilt series was acquired of $K$. pneumoniae under brightfield TEM a, where multiple nanopillars appeared to be penetrating the envelope (1-6). Analysis of nanopillar tip coordinates in $x, y$ and $z$ revealed that nanopillars 1-4 and 6 had penetrated the envelope, while nanopillar 5 was only deforming the cell. A 3D reconstruction of the tomogram revealed all nanopillars had penetrated the bacterial envelope by at least $100 \mathrm{~nm} \mathbf{b}$. The dark bands seen within nanopillars are bend contours; these spatial contrasts arise from local bending or deformation of nanopillars. Electron tomography was performed on five $K$. pneumoniae cells interacting with $\mathrm{TiO}_{2}$ nanopillars, these were acquired in four tilt series.

Cross sectional analysis was also performed on $S$. aureus in contact with three nanopillars (Fig. 7). Analysis of individual FIB cross sections confirmed that nanopillar 2 had penetrated the bacterial envelope by $48 \mathrm{~nm}$, surpassing the envelope by $\sim 15 \mathrm{~nm}$. Thus, in contrast to $E$. coli, where nanopillar 1 had induced significant local deformation, in S. aureus, envelope penetration did not appear to affect cell morphology. These findings corroborate our cross sectional analysis using TEM, which provides evidence that nanopillars had penetrated the envelope of $S$. aureus without significant alterations to morphology. 
a

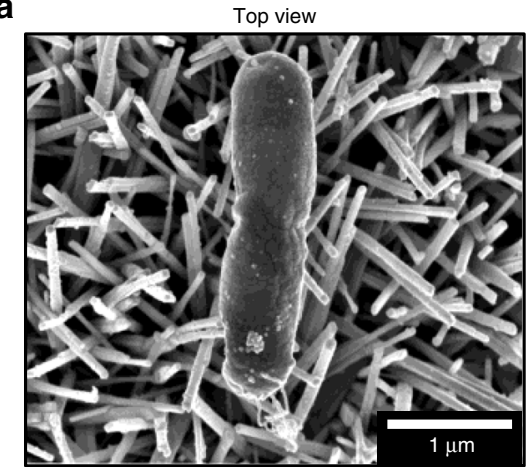

d

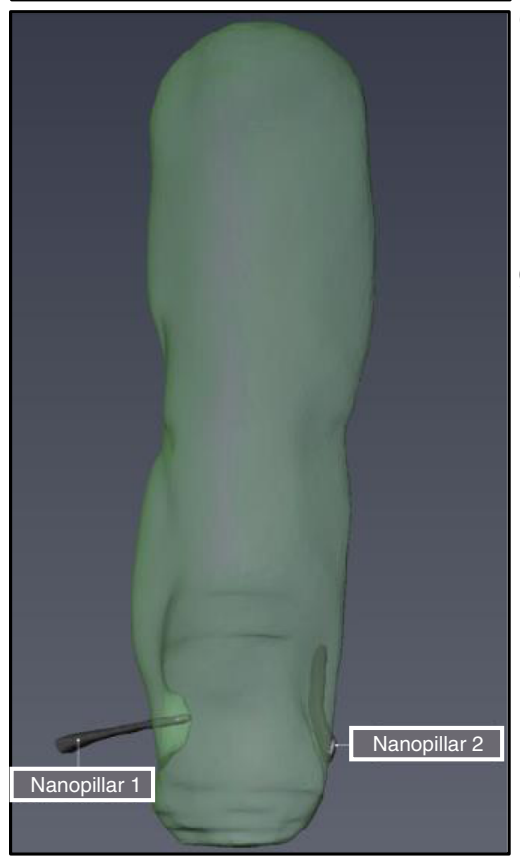

b
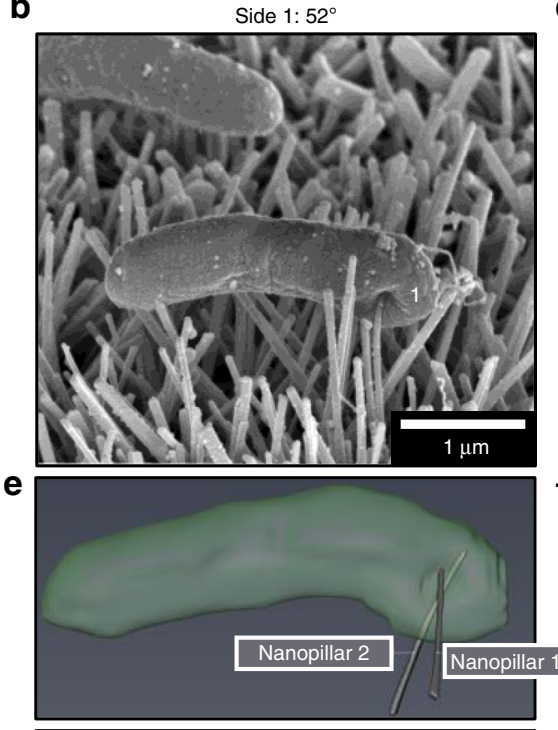

$\mathbf{g}$

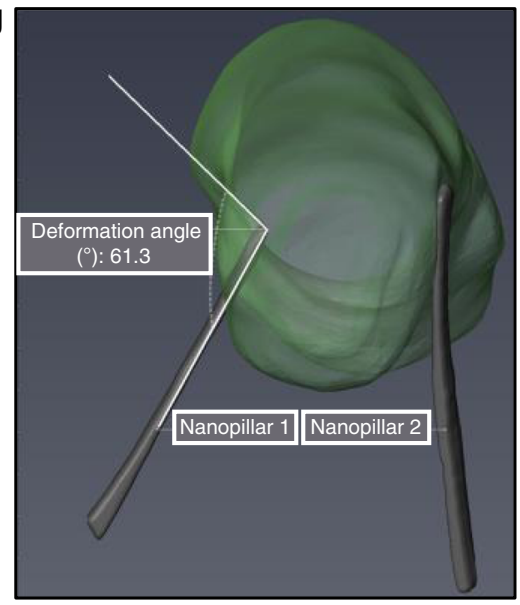

C
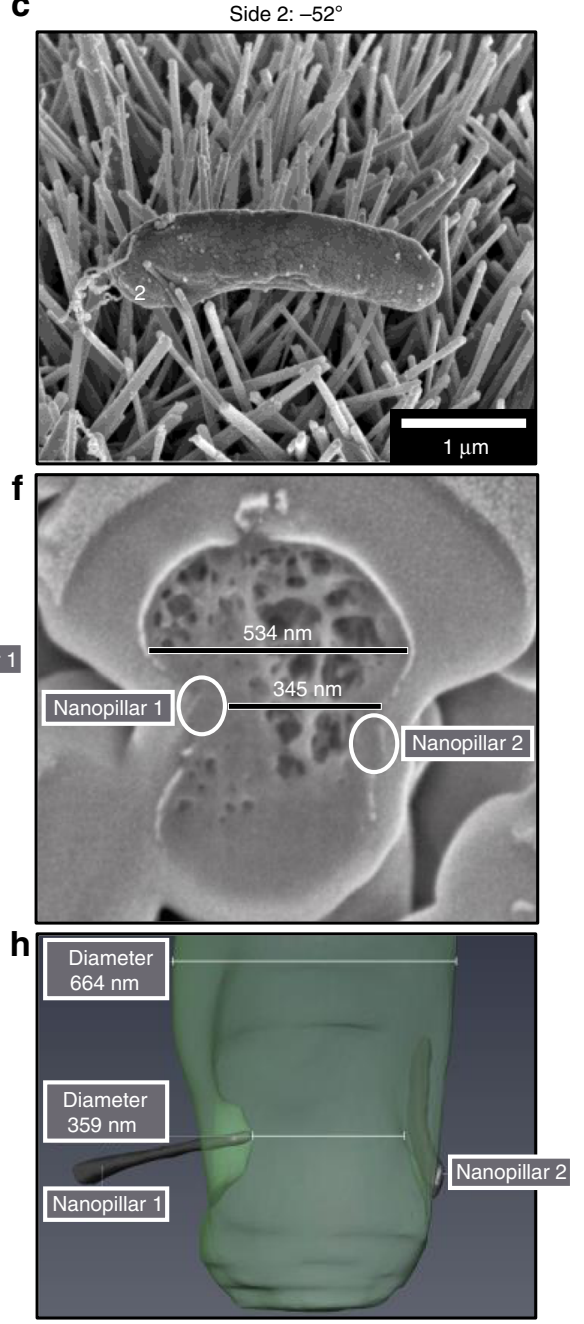

Fig. 6 3D FIB-SEM reconstruction of E. coli. Automated FIB-SEM cross sectional analysis was performed on an $E$. coli cell a that was pinned between two nanopillars b, c. The focused ion beam produced 120 cross sections ( $30 \mathrm{~nm}$ each) that were imaged, and reconstructed in Avizo d, e. Analysis of $E$. coli cross section \#30 showed that nanopillar 1 had forced E. coli into nanopillar 2, resulting in a $189 \mathrm{~nm}$ reduction in cell width $\mathbf{f}$. While nanopillar 1 had induced significant envelope deformation, no evidence of envelope penetration was found, and the width of $E$. coli cell quickly restored to normal either side of the contact point, indicating that the cell had not lost turgor pressure $\mathbf{g}$, h. FIB-SEM analysis was performed on four E. coli cells interacting with $\mathrm{TiO}_{2}$ nanopillars.

$\mathrm{TiO}_{2}$ nanopillars impair cell viability. The observations of nanopillar-induced envelope deformation and penetration in both Gram-positive and Gram-negative bacteria, led us to consider that nanopillars may have affected cell viability. The antibacterial activity of NW-850-5 compared to control titanium surfaces was initially assessed using the luminescent metabolic indicator assay RealTime-Glo. Growth of S. aureus, E. coli or K. pneumoniae was monitored in situ by recording luminescence in a temperature-controlled $\left(37^{\circ} \mathrm{C}\right)$ plate reader for $10 \mathrm{~h}$ (Fig. 8). The rate of luminescence production by $S$. aureus was noticeably slower on NW-850-5 and plateaued after $6 \mathrm{~h}$, while on controls, the signal increased over the entire 10 -h incubation period. Levels of metabolic activity differed significantly from controls on NW850-5 after 9 h (Fig. 8a). Similar trends were observed for E. coli, although the differences between nanopillar and control surfaces did not reach statistical significance (Fig. 8b). A contributory factor to this was that the absolute relative luminescence unit (RLU) values were significantly (1000-fold) lower for E. coli than for $S$. aureus, which most likely reflects the lower reducing capacity of Gram-negative bacteria. This was further supported when K. pneumoniae was tested. The absolute RLU values were much lower and no significant differences were observed, although the luminescence profile for NW-850-5 was distinct from the other two surfaces (Fig. 8c). Of note, E. coli and $K$. pneumoniae studies showed a reduction in luminescence signal on controls from 6 and $5 \mathrm{~h}$, respectively. This may indicate that other factors, independent of the surface, were affecting growth at these times.

Given the low sensitivity of RealTime-Glo with Gram-negative bacteria in this study, viability testing was additionally performed using endpoint assay BacTiter-Glo, and luminescence data were converted to bacterial CFU based on standard curves (Supplementary Fig. 9). Contrary to RealTime-Glo analyses, BacTiterGlo revealed significant differences in $E$. coli viability on NW850-5 following 3- and 10-h incubations relative to controls. Similarly, BacTiter-Glo revealed significant differences in $K$. pneumoniae viability on NW-850-5 following 3-h incubations relative to controls; although significance was not reached at $10 \mathrm{~h}$ (Fig. 8c). For S. aureus, significant reductions in CFU relative to controls were observed on NW-850-5 following 10-h incubation (Fig. 8a), corroborating the RealTime-Glo data.

Bacterial proteomic response to nanopillar surface NW-850-5. SEM determination of bacterial densities (Supplementary Figs. 6-8) 
a

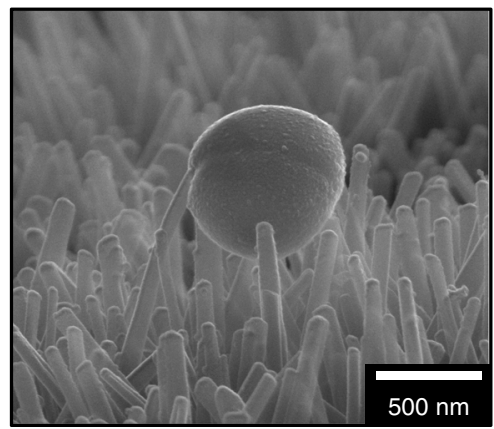

d

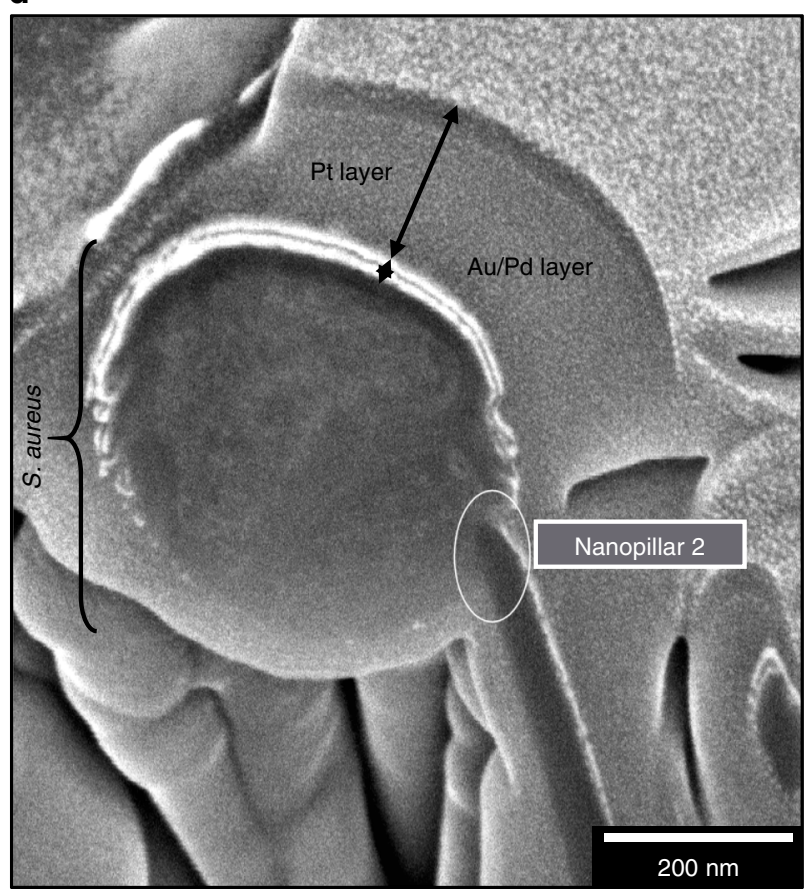

b

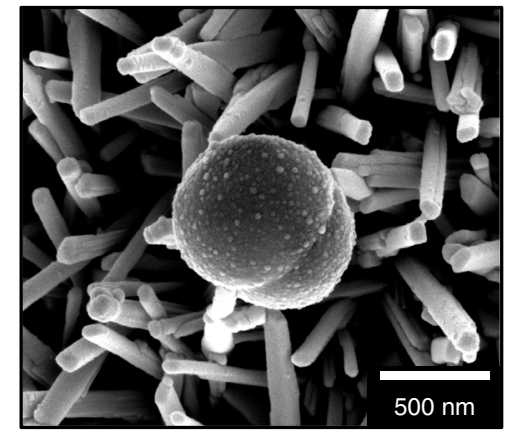

C

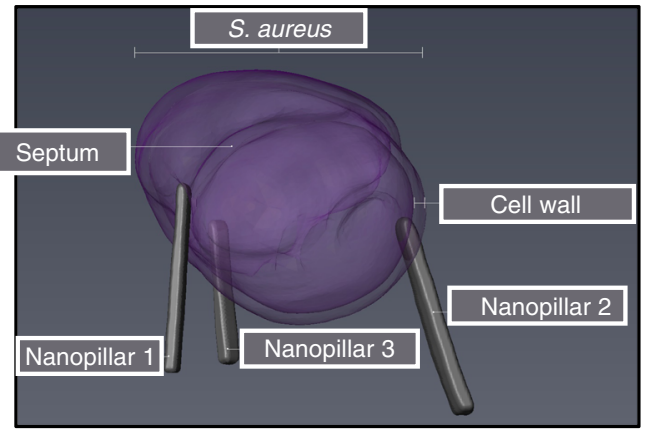

e

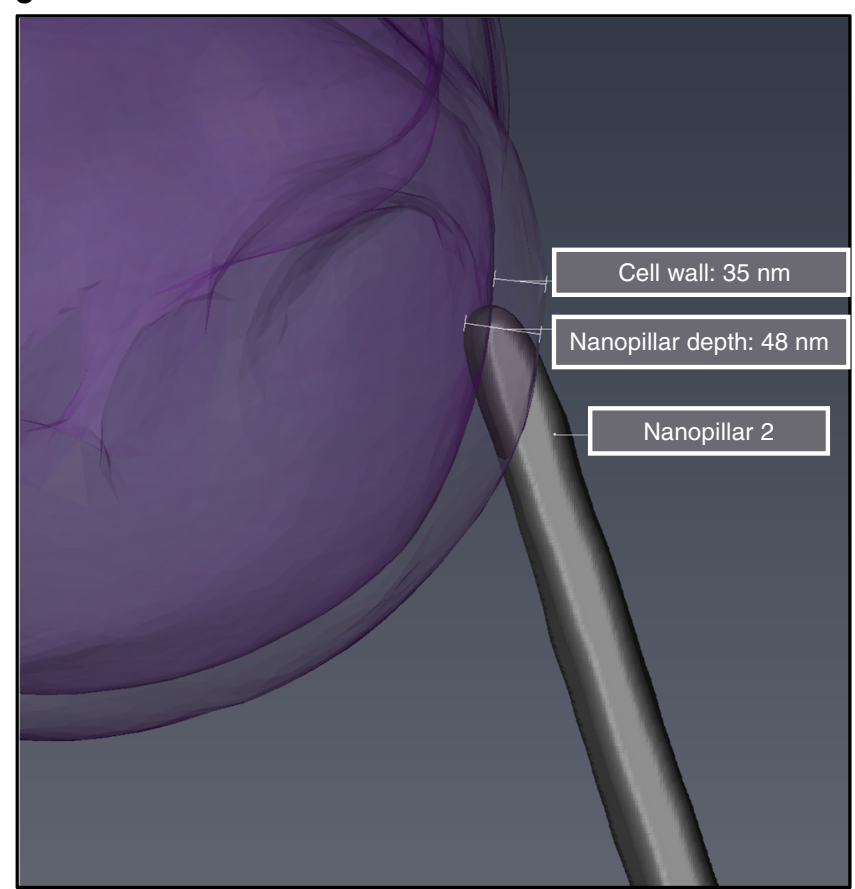

Fig. 7 3D FIB-SEM reconstruction of S. aureus. Automated FIB-SEM cross sectional analysis was performed on an S. aureus cell interacting with three nanopillars a, b. The focused ion beam produced 57 cross sections ( $30 \mathrm{~nm}$ each) that were imaged, and reconstructed in Avizo c. Analysis of $S$. aureus cross section \#40 showed the tip of nanopillar 2 located $\sim 50 \mathrm{~nm}$ into the cell, providing confirmation of cell wall and inner envelope penetration d, e. FIBSEM analysis was performed on one $S$. aureus cell interacting with $\mathrm{TiO}_{2}$ nanopillars.

and bacterial viability analysis (Fig. 8) confirmed that nanopillars had impaired bacterial growth. However, the relatively low frequency with which envelope deformation and penetration were observed could not account for the concomitant reductions in bacterial viability seen on nanowire arrays relative to controls. It was considered then that nanopillars might trigger a physiological response ${ }^{19-21}$, reducing the capacity of bacteria to proliferate. To explore this hypothesis, TMT labelling and mass spectrometry analysis were performed to enable a direct quantitative comparison of $S$. aureus and E. coli proteomes after $24 \mathrm{~h}$ in the presence or absence of NW-850-5. Proteomic analysis revealed that $90 \mathrm{~S}$. aureus proteins and $27 \mathrm{E}$. coli proteins exhibited differential expression with a $P \leq 0.05$, corresponding to 7 and $2 \%$ of the total proteomes, respectively (Supplementary Figs. 10a and 11a). Gene Ontology (GO) enrichment analysis was used to categorise each protein by molecular function, biological process and cellular component (Supplementary Figs. 10b and 11b). To uncover whether $S$. aureus and $E$. coli differentially expressed proteins (DEPs) were biologically connected, protein-protein interactions were mapped using the STRING application within Cytoscape (Fig. 9) ${ }^{26,27}$. In both instances, the number of interactions
(S. aureus, $421 ;$ E. coli, 14) was significantly higher than would be expected for a random set of proteins of similar size drawn from the genome. This verified that the $S$. aureus and E. coli DEP networks were biologically connected.

A number of the $S$. aureus DEPs are associated with protection from oxidative stress. Of note, superoxide dismutase, responsible for the conversion of superoxide anions into oxygen and hydrogen peroxide $\left(\mathrm{H}_{2} \mathrm{O}_{2}\right)^{28}$, had increased over two-fold in $S$. aureus recovered from NW-850-5. The abundance of other oxidative stress proteins had also increased in $S$. aureus, namely methionine sulfoxide reductase, responsible for repairing the oxidation of methionine residues ${ }^{29}$, and bacterioferritin comigatory protein, a thiol peroxidase involved in the reduction of $\mathrm{H}_{2} \mathrm{O}_{2}$ (ref. ${ }^{30}$ ). Furthermore, the NADH-dependent flavin oxidoreductase (NWMN_0315) had increased nearly six-fold in the presence of NW-850-5. Expression of such proteins is induced by $\mathrm{H}_{2} \mathrm{O}_{2}$ stress $^{31}$. SOS response proteins UvrA and UvrC, and bleomycin resistance protein had also increased significantly in the presence of NW-850-5, which mediate repair of DNA damage ${ }^{32,33}$, along with histone-like DNA-binding protein $\mathrm{HU}$, which is involved in DNA stabilisation under extreme environmental conditions ${ }^{34}$. In 

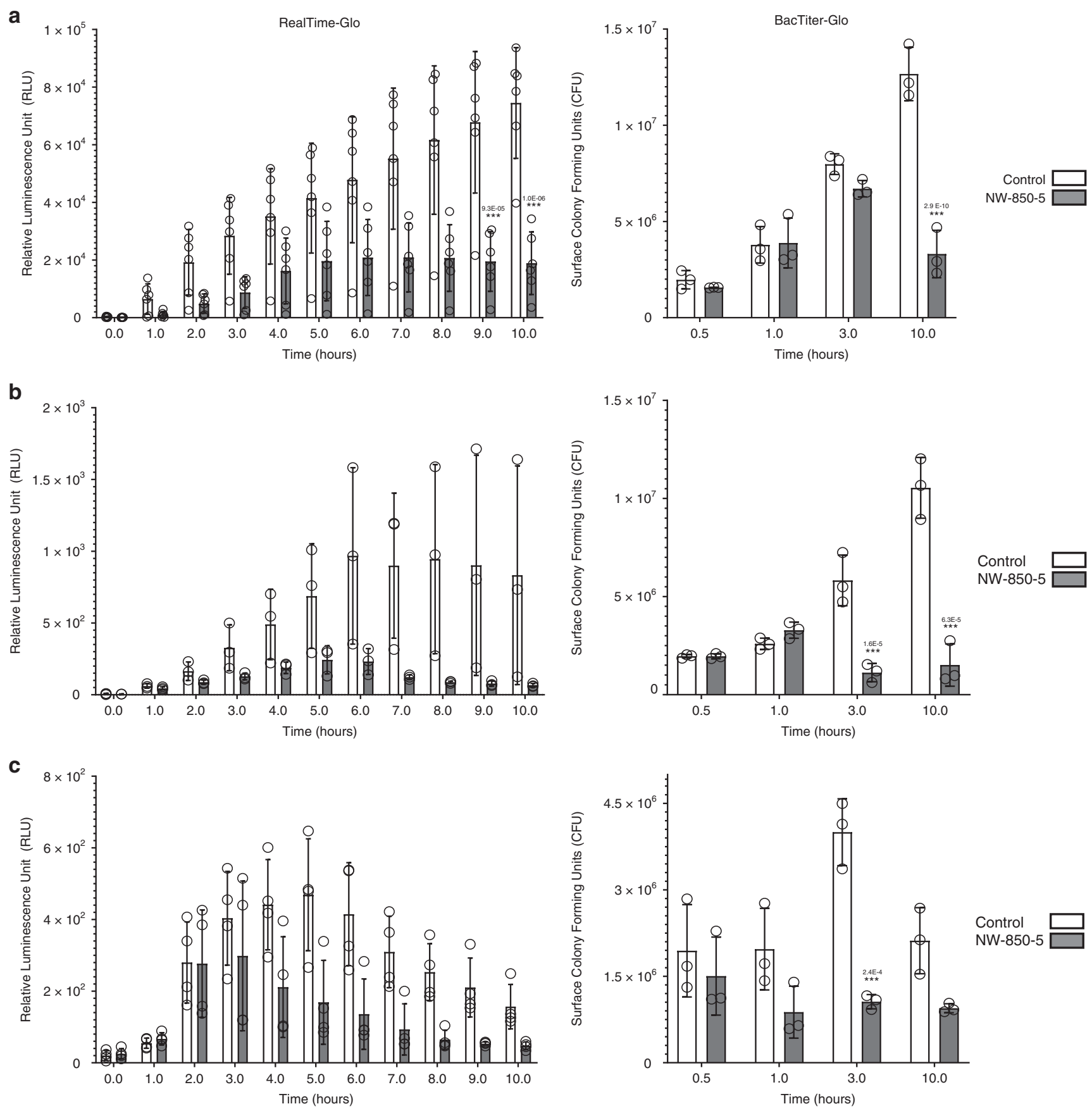

Fig. 8 Determining bacterial viability on nanopillar surfaces. Luminescence signals and CFU of $S$. aureus $\mathbf{a}$, E. coli $\mathbf{b}$ and $K$. pneumoniae $\mathbf{c}$ incubated on control or nanopillar $\mathrm{TiO}_{2}$ surfaces for up to $10 \mathrm{~h}$, as determined by real-time or endpoint assays, respectively. Values are given as mean \pm standard deviation and individual data points for each mean are shown. ${ }^{\star \star *}$ indicates $P \leq 0.001$ relative to control, as determined by one-way ANOVA and Tukey HSD post hoc test. BacTiter-Glo experiments $n=3$; RealTime-Glo experiments $n=6$ (S. aureus), $n=3$ (E. coli) and $n=4$ (K. pneumoniae). Total surface CFU were determined from disc areas of $0.64 \mathrm{~cm}^{2}$. Exact $P$-values are indicated for each significant time point.

addition to oxidative stress, another key theme of $S$. aureus DEPs was protein expression. A significant proportion (33\%) of DEPs were involved in protein translation, secretion and translocation, all of which had been upregulated in the presence of NW-850-5.

As for $S$. aureus, oxidative stress was a key theme of the DEPs identified for E. coli. Subunit A (ClpA) of the ATP-dependent protease (ClpAP), which mediates unfolding and translocation of abnormal proteins ${ }^{35}$, had decreased significantly in the presence of NW-850-5. Oxidative stress is known to inactivate such chaperone proteins ${ }^{36}$. Concomitantly, chaperedoxin had increased in the presence of NW-850-5, which is known to protect against the irreversible protein aggregation and oxidation $^{36}$. Another notable E. coli DEP was surface composition regulator GlgS. This is a negative regulator of type-1 fimbriae and flagella production ${ }^{37}$, and was increased significantly in E. coli recovered from NW-850-5. It is possible that the increased abundance of GlgS could account for the absence of extracellular appendages observed for $E$. coli on NW-850-5 under SEM. Nanoscale variations in surface topography are reported to significantly alter protein expression in bacteria, and Rizzello and colleagues found that $E$. coli adhered to gold nanoparticle 

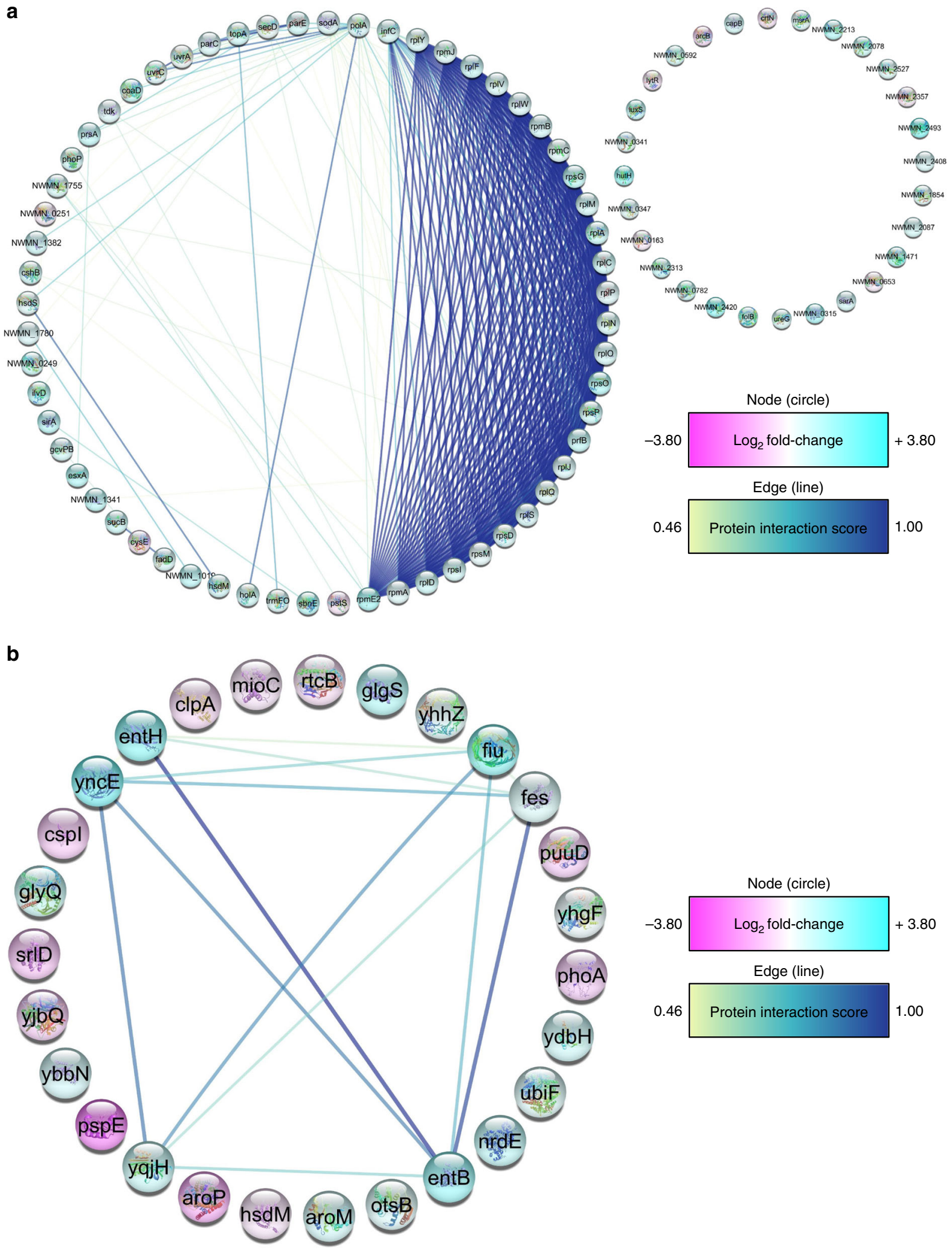

Fig. 9 S. aureus and $\mathbf{E}$. coli proteomic response to nanopillar surface $\mathbf{N W - 8 5 0 - 5}$. The predicated functional partners among S. aureus a and $E$. coli b DEPS are shown, where individual proteins are represented in circles (nodes), and protein-protein interactions (PPI) are represented by the connecting lines (edges). DEPs have been coloured according to the log2 fold change in protein abundance (pink = decreased abundance and blue $=$ increased abundance), and node colour indicates the confidence of supporting interaction evidence $(0.15=$ low confidence, $0.40=$ medium confidence, $0.70=$ high confidence and 0.9 =highest confidence). For S. aureus, 60 DEPs displayed at least one interaction at a confidence level between 0.46 and 1.00 , and the PPI enrichment $P$-value was $1.0 \mathrm{e}-16$. For $E$. coli, six proteins had at least one interaction at a medium to high confidence and had a PPI enrichment $P$-value of 9.54e-06. PPI enrichment $P$-values were determined using a hypergeometric test and corrected for multiple testing using the method of Benjamini and Hochber ${ }^{54}$. Data are representative of one experimental replicate, performed in triplicate. 
surfaces expressed significantly lower levels of type-1 fimbriae. Concomitantly, proteins involved in DNA and membrane stress were upregulated $^{19}$.

Determination of oxidative stress response to $\mathrm{NW}-850-5$. Taken together, proteomic analysis, while preliminary, suggested that $\mathrm{TiO}_{2}$ nanopillars had induced an oxidative stress response within $S$. aureus and E. coli. A possible explanation for oxidative stress could be increased reactive oxygen species (ROS), as has been reported on CNT surfaces ${ }^{38}$. To investigate this as a possible mechanism for reduced viability, the ROS-Glo assay was used to measure the levels of $\mathrm{H}_{2} \mathrm{O}_{2}$ (an oxidative stress marker) produced from $S$. aureus and E. coli, following 24-h incubation on NW850-5 or control discs. The levels of $\mathrm{H}_{2} \mathrm{O}_{2}$ in S. aureus and E. coli culture media were significantly higher on NW-850-5 compared to control discs, corresponding to 1.6- and 3.8-fold increases, respectively (Fig. 10a). Given that ROS mediate damage of DNA, lipids and proteins ${ }^{32,39}$, the increased quantity of $\mathrm{H}_{2} \mathrm{O}_{2}$ could be expected to induce changes in bacterial envelope morphology. Following 24-h incubation, the density and coverage of $S$. aureus and E. coli were notably higher on control compared to NW-8505 discs (Fig. 10). At higher magnification, the characteristic morphologies of S. aureus (coccoid) and E. coli (bacillus) were observed on control surfaces (Fig. 10b, d), with evidence of microcolony and biofilm formation. In contrast, the morphology of $S$. aureus and E. coli on NW-850-5 discs was less rigid and did not conform to the defined shapes observed on control discs (Fig. 10c, e). This was particularly evident for E. coli, which appeared to be sinking into the nanopillars. These morphologies were consistent with previous studies that have proposed ROSmediated cell death on CNTs (ref. ${ }^{38}$ ).

\section{Discussion}

Elucidating the mechanisms that govern bacterial cell death on nanotextured materials is essential for improving antibacterial performance. This, in turn, will guide the design of nextgeneration biomaterials that effectively inhibit biofilm growth, without reliance on antibiotics. To date, natural and synthetic nanotextured materials are reported to mediate bactericidal activity via mechanical rupture of the bacterial cell, resulting in lysis and cell death ${ }^{1-6}$. In this study, analysis of bacterial cross sections using TEM and tomography identified clear signs of localised nanopillar-induced envelope deformation and penetration. However, there was no evidence to support that bacterial lysis had occurred, even with the concomitant reductions in bacterial viability. Similar cases of nanopillar-induced envelope deformation and penetration were identified by FIB cross sectional analysis. These observations were most prominent in Gram-negative bacteria E. coli and K. pneumoniae, which possess thin peptidoglycan layers $(\approx 5 \mathrm{~nm})$. Nonetheless, there was strong evidence that nanopillars were also able to penetrate $S$. aureus, albeit at a much lower frequency. The reduced susceptibility of $S$. aureus to nanopillar deformation may partly be explained by the increased peptidoglycan thickness, providing increased rigidity ${ }^{6}$ and higher turgor pressure; indeed, one study reported a lower total creep deformation in B. subtilis compared to E. colito. In another study, the force required to rupture Staphylococcus epidermidis was nearly four-fold greater than for E. coli, measured at $13.8 \mu \mathrm{N}$ and $3.6 \mu \mathrm{N}$, respectively ${ }^{41}$. Although $S$. aureus appeared less susceptible to nanopillar-induced killing than $E$. coli or $K$. pneumoniae, further investigations are required to assess the antibacterial properties of nanopillar surfaces over longer incubations and to establish their performance against microorganisms with thicker cell walls (e.g., fungi and spores).
Although envelope deformation and penetration were confirmed in this study, the frequency of such events was low and did not result in mechanical rupture and cell lysis. Therefore, these interactions could not solely account for the reductions in cell viability that were recorded. However, proteomic analyses identified a number of $S$. aureus and E. coli DEPs associated with oxidative stress. This was further supported by ROS-Glo analysis, which revealed that $\mathrm{H}_{2} \mathrm{O}_{2}$ levels were significantly higher in $S$. aureus and $E$. coli incubated on NW-850-5 than on controls. Bacterial ROS production is frequently observed following exposure to bactericidal antibiotics ${ }^{42-45}$. This mechanism is reported to be self-amplifying, meaning that ROS production is sustained, even when the initial stressor is removed ${ }^{39}$. Bacterial oxidative stress may also be activated by other stressors, such as copper $^{46}$ or the accumulation of misfolded proteins ${ }^{28}$. Given that bacterial oxidative stress has overlapping activating signals ${ }^{47}$, it is possible that the increased abundance of oxidative stress proteins and elevated levels of $\mathrm{H}_{2} \mathrm{O}_{2}$ reported here are directly attributable to the effects of the $\mathrm{TiO}_{2}$ nanopillars.

Previous research has established a connection between ROSmediated bacterial cell death and nanostructure contact. In one study, CNTs were shown to increase ROS production in Candida albicans, $P$. aeruginosa and $S$. aureus following 24-h incubation. ROS production was proposed to have been induced by physical contact with the CNTs, which were reported to wrap around bacteria rather than pierce the envelope. Of note, ROS production was shown to occur independently of photocatalytic activation ${ }^{38}$. No photocatalytic activity was detected on the $\mathrm{TiO}_{2}$ nanopillar surfaces in this study, as the luminescence generated by the ROSGlo assay was negligible in the absence of bacteria. This is most likely attributable to the rutile crystal structure of $\mathrm{TiO}_{2}$ nanopillars, which generally possesses lower photocatalytic activity than anatase polymorphs ${ }^{48,49}$.

This study has highlighted several key findings that have important implications for the development of antimicrobial nanotopographies for biomedical applications. Firstly, the mechanistic basis of contact killing is multifactorial (Supplementary Fig. 12) and nanotopography dependent. While deformation and subsequent penetration of the bacterial envelope by nanopillars was confirmed in this study, these mechanisms did not result in mechanical rupture or cell lysis. Another notable mechanism identified in this study was nanopillar-induced cell impedance, which is expected to reduce the capacity of bacteria to replicate on nanopillar surfaces, and thus could enhance the antibiofilm properties of nanopillar surfaces. Furthermore, our analyses provide compelling evidence that nanopillars can induce oxidative stress within bacterial cells upon contact, the cumulative effects of which could impair processes, such as bacterial growth and biofilm formation. Indeed, this could account for the timedependent reductions in bacterial viability. Better understanding of this mechanism could prove invaluable for improving the antibacterial performance of nanotextured materials. Thus, further research is needed to examine more closely the link between ROS generation, nanotopography design and bactericidal activity. It is also evident from this study that multiple experimental approaches should be exploited to robustly assess all aspects of bacterial physiology, so as to obtain a comprehensive assessment of the antibacterial properties of nanotextured materials. This will reduce experimental bias toward a single mechanism of action, and provide a more complete and accurate understanding of the antimicrobial mechanism of nanotopographies.

\section{Methods}

Generation of $\mathrm{TiO}_{2}$ nanopillars on titanium by thermal oxidation. Grade 5 titanium alloy (Ti-6Al-4V, Titanium Metals Ltd) samples $\left(0.64 \mathrm{~cm}^{2}\right)$ were machine polished (Struers ${ }^{\circledR}$ TegraForce-1) using decreasing silicon carbide grit sizes ( $\# 80$, 
a

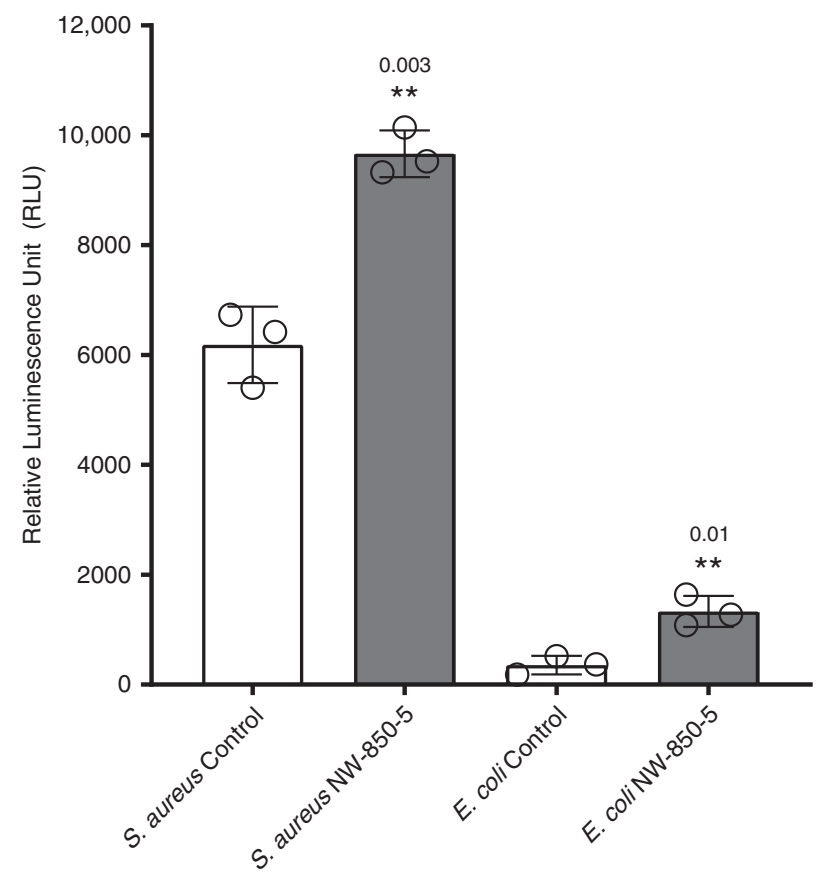

b

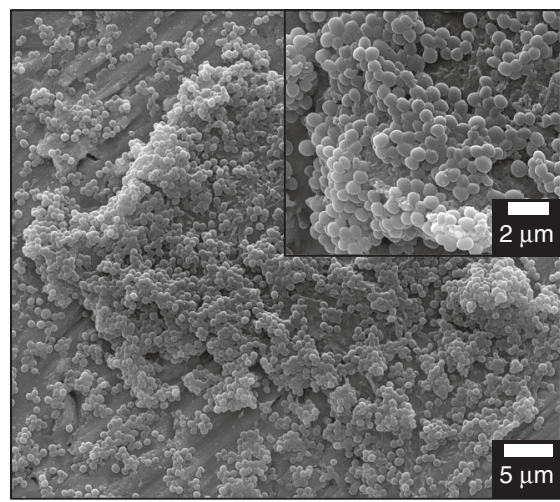

C

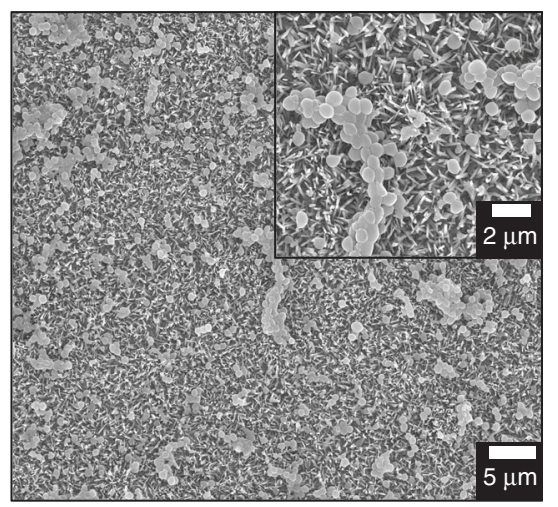

d

e
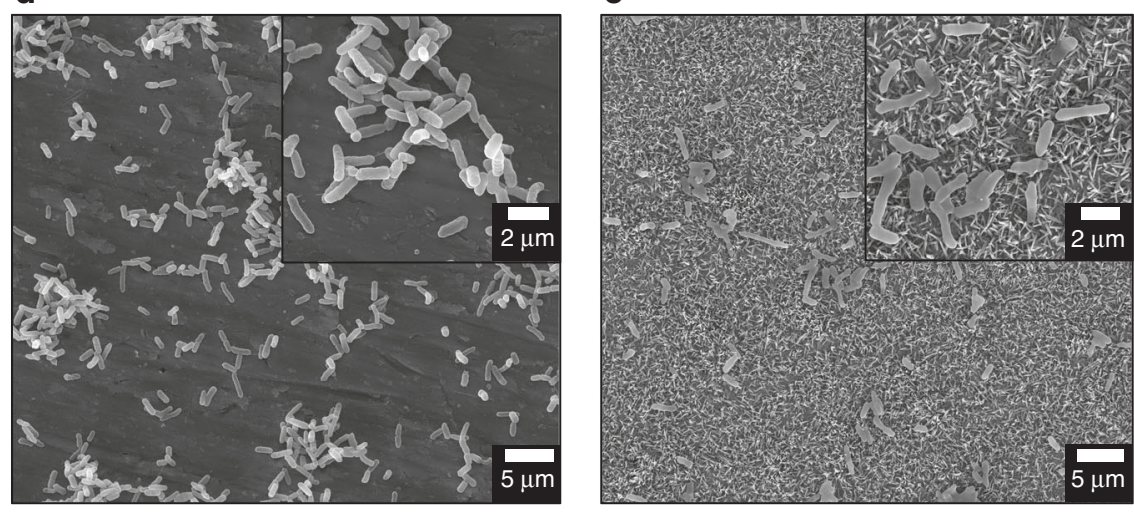

Fig. 10 Determination of ROS production in response to nanopillar surfaces. S. aureus or E. coli were incubated for $24 \mathrm{~h}$ on control or NW-850-5 surfaces under the same growth conditions used for proteomic analysis. Following 24-h incubation, the levels of $\mathrm{H}_{2} \mathrm{O}_{2}$ were determined by ROS-Glo a. Values given are mean \pm standard deviation and individual data points for each mean are shown. ${ }^{\star}$ indicates $P \leq 0.01$ relative to control for $S$. aureus $(P=0.003)$ and E. coli $(P=0.01)$, as determined by a two-sided Student's $t$-test; $n=3$ performed in duplicate. Representative SEM micrographs of $S$. aureus $\mathbf{b}, \mathbf{c}$ and $E$. coli d, e incubated on control or NW-850-5 are shown. Insets show higher magnification SEM images of each surface.

\#500, \#1200, \#2000 and \#4000). To remove surface contaminants, titanium discs were placed inside a digital ultrasonic bath (Grant Scientific XUB series) in distilled water $\left(\mathrm{dH}_{2} \mathrm{O}\right)$, and the samples were cleaned at $40{ }^{\circ} \mathrm{C}$ for 15 min using $100 \%$ power. Following ultrasonication, titanium samples were placed in ethanol (analytical reagent grade (99.99\%), Fisher Scientific) for 10 min before air drying. Polished and cleaned titanium samples were sealed inside a horizontal alumina tube $(120 \mathrm{~cm} \times$ $11 \mathrm{~cm}$ outer $\times 9 \mathrm{~cm}$ inner) positioned in a furnace (Elite Thermal Systems Ltd). Prior to thermal oxidation, the furnace was purged with inert argon gas (Ar) to remove oxygen and achieve a one-directional flow. Following purging, heating was initiated at $15^{\circ} \mathrm{C}$ per minute until a pre-defined maximum was reached; in this 
study, a temperature of $850{ }^{\circ} \mathrm{C}$ was used. Once the final temperature was reached, Ar was redirected into a sealed Duran ${ }^{\mathrm{TM}}$ vessel containing liquid acetone (analytical reagent grade $(99.99 \%)$, Fisher Scientific), maintained at $25^{\circ} \mathrm{C}$. This generated an acetone vapour phase, which initiates the oxidation reaction with titanium samples. Following completion of the heating programme, furnace cooling began. The flow of Ar was maintained at a constant rate until room temperature was reached.

\section{Nanopillar characterisation. A Quanta 200 field emission gun (FEG) SEM (FEI)} was used to determine the dimensions of nanopillars produced by thermal oxidation. To establish the homogeneity of nanopillar coverage and the reproducibility between thermal oxidation batches, top view electron micrographs were taken of discs from three independent batches; micrographs were taken at three locations per disc. FIJI software was used to quantify the density of nanopillars per $\mu \mathrm{m}^{2}$, using the find maxima function. Tilted $\left(40^{\circ}\right)$ electron micrographs were used to estimate the length and tip diameter of nanopillars, with three locations captured per disc, from three independent batches. A total of 20 length and 20 diameter measurements were recorded from each micrograph. These data were averaged from each batch to estimate the mean nanopillar length and diameter. The elemental composition of nanopillars was determined using EDX in line-scanning mode on a JEOL JEM-2100F FEG TEM. The crystal structure of $\mathrm{TiO}_{2}$ nanopillars was analysed with grazing-incident X-ray diffraction. Titanium samples were mounted $0.243 \mathrm{~m}$ from the detector and tilted at $0.7^{\circ}$. An X-ray beam (wavelength of $885.6 \mathrm{~nm}$ ) was shot at the sample and the diffracted X-rays were detected and imaged at two positions, $8^{\circ}$ and $30^{\circ}$. The images were converted into $1 \mathrm{D}$ profiles by azimuthal integration using a pyFAI package. The ratio of anatase to rutile was determined using the Spurr and Myers equation ${ }^{50}$, by calculating the area underneath characteristic anatase $(2 \theta=26.5)$ and rutile $(2 \theta=27.5)$ peaks.

\section{Bacterial strains and culture conditions. S. aureus strain Newman, S. aureus} SH1000 (ref. ${ }^{51}$ ), E. coli strain K12, E. coli TOP10 (ref. ${ }^{52}$ ) and K. pneumoniae (clinical isolate kindly provided by $\mathrm{M}$. Avison) were used in this study. Broth cultures were incubated for $16 \mathrm{~h}$ at $37^{\circ} \mathrm{C}, 220 \mathrm{rpm}$, subcultured to $\mathrm{OD}_{600} 0.1$, and grown to mid-exponential phase. Bacteria were routinely cultured in Luria Bertani broth (BD Biosciences) apart from for the BacTiter-Glo assay, for which Mueller Hinton broth (Sigma-Aldrich) was used. Titanium samples were sterilised in absolute ethanol, washed in $\mathrm{dH}_{2} \mathrm{O}$ and dried prior to inoculation with bacterial suspensions. For all bacterial viability assays, titanium discs were incubated statically at $37^{\circ} \mathrm{C}$.

Determination of bacterial viability. For BacTiter-Glo analysis, sterile titanium discs were placed inside white 24-well plates (PerkinElmer, MA, USA) and inoculated with $25 \mu \mathrm{L}$ of bacterial culture (equivalent to $10^{6}-10^{7} \mathrm{CFU}$ ), forming a meniscus. Titanium discs were incubated for $0.5,1,3$ and 10 -h time points at $37^{\circ} \mathrm{C}$ under static conditions inside a humidity chamber. Following incubation, equilibrated BacTiter-Glo reagent was added in a 1:1 ratio to the bacterial suspension and incubated for $5 \mathrm{~min}$ in the dark, prior to RLU measurements. Quantification of the total number of bacterial cells on each titanium disc were based on standard curves relating RLU to CFU (Supplementary Fig. 9). For RealTime-Glo assays, sterile titanium discs were inoculated with $50 \mu \mathrm{L}$ of bacterial culture (equivalent to $\left.10^{6}-10^{7} \mathrm{CFU}\right)$, supplemented with $1 \times$ MT Cell Viability Substrate and $1 \times$ NanoLuc ${ }^{\circledast}$ Enzyme. Each 24-well plate was sealed and luminescence measurements were recorded continuously for $10 \mathrm{~h}$ at $37^{\circ} \mathrm{C}$ under static growth conditions. For both assays, luminescence was recorded using an automated Infinite ${ }^{\circledast} 200$ PRO microplate reader (Tecan, Männedorf, Switzerland). Luminescence was recorded using automatic attenuation, $1 \mathrm{~s}$ integration time and $0.15 \mathrm{~s}$ settle time.

SEM sample preparation and imaging. Bacteria on titanium discs were fixed at $4{ }^{\circ} \mathrm{C}$ in $2.5 \%$ EM grade glutaraldehyde (Agar Scientific Ltd. Essex, UK) buffered with $0.1 \mathrm{M}$ sodium cacodylate (Acros Organics, New Jersey, USA). Following overnight fixation, samples were washed $(3 \times 5 \mathrm{~min})$ in $0.1 \mathrm{M}$ sodium cacodylate buffer and dehydrated in a graded ethanol series of $25 \%, 50 \%, 70 \%, 90$ and $100 \%$ (Sigma-Aldrich, St. Louis, MO, USA). Samples were then critically point dried using a Leica CPD300, following an established protocol for microbial cells ${ }^{53}$. Prior to image acquisition on a Quanta 200 FEG-SEM, titanium discs were mounted to 0.5 " aluminium stubs using colloidal silver paste (Agar Scientific Ltd. Essex, UK), before being coated with a $10 \mathrm{~nm}$ chromium layer using an Emitech K757X sputter coater system.

Sample preparation for TEM and FIB-SEM. Following overnight fixation in $2.5 \%$ EM grade glutaraldehyde at $4{ }^{\circ} \mathrm{C}$, samples were washed $(3 \times 5 \mathrm{~min})$ in $0.1 \mathrm{M}$ sodium cacodylate buffer prior to OTO (osmium

tetraoxide-thiocarbohydrazide-osmium) processing. Briefly, this method included post fixation in equal volumes of $4 \%$ osmium tetraoxide (Agar Scientific Ltd. Essex, UK) and 3\% potassium ferrocyanide (Sigma-Aldrich, St. Louis, MO, USA) for $60 \mathrm{~min}$ on ice. Following post fixation, samples were rinsed $(3 \times 5 \mathrm{~min})$ in $\mathrm{dH}_{2} \mathrm{O}$ before incubating with thiocarbohydrazide (Sigma-Aldrich, St. Louis, MO, USA) for $20 \mathrm{~min}$. Additional $\mathrm{dH}_{2} \mathrm{O}$ washing steps $(3 \times 5 \mathrm{~min})$ were applied before incubation in $2 \%$ aqueous osmium for $30 \mathrm{~min}$ at room temperature. Following
OTO processing, bacterial samples were stained in $1 \%$ aqueous uranyl acetate $(1 \mathrm{~h}$ at $\left.4{ }^{\circ} \mathrm{C}\right)$ followed by lead aspartate $(200 \mu \mathrm{M})$ for $30 \mathrm{~min}$ in the dark. Between these steps, washing with $\mathrm{dH}_{2} \mathrm{O}$ was performed. After the final washing step, bacterial samples were dehydrated and dried as previously outlined.

TEM sectioning and imaging. Bacteria incubated on control titanium discs were recovered and pelleted prior to staining and microtomy, whereas bacteria incubated on $\mathrm{TiO}_{2}$ nanopillar surfaces were stained, embedded and sectioned, while attached to the titanium disc. All samples to be analysed using TEM were embedded in epon 812 resin (TAAB Laboratories Equipment Ltd. Berkshire, UK) for $90 \mathrm{~min}$ on a TAAB rotator type N, held at $2 \mathrm{rpm}$. Samples were then placed at $60^{\circ} \mathrm{C}$ for $48 \mathrm{~h}$ to allow resin polymerisation. Resin embedded samples were sectioned using an Ultra Diamond Knife (DiATOME) on a Leica EM UC6 ultramicrotome. These were transferred to pioloform-coated TEM grids for microscopy. To analyse the ultrastructure of bacterial cells adhered to $\mathrm{TiO}_{2}$ nanopillar surfaces, samples were loaded into a Tecnai 12 FEI $120 \mathrm{kV}$ BioTwin Spirit TEM. An electron beam with an accelerating voltage of $120 \mathrm{kV}$ was used to capture images. For tilt series acquisition, TEM samples were transferred to a Fischione tomography holder and loaded into a Tecnai 20-FEI $200 \mathrm{kV}$ Twin Lens scanning TEM. Tilt series projections were acquired in bright-field mode. Dedicated FEI tomography software was used to capture the tilt series data. An electron beam with an accelerating voltage of $200 \mathrm{kV}$ was used to capture images.

FIB sectioning and imaging. Titanium samples were loaded into the vacuum chamber of a Strata FIB201 (FEI). When searching for bacteria of interest, an electron beam with an accelerating voltage of $5 \mathrm{kV}$ and current of $98 \mathrm{pA}$ was used. To preserve bacterial morphology, electron and ion-assisted platinum deposition was performed at $27.5 \mathrm{pA}$, creating a protective layer $0.5 \mu \mathrm{m}$ thick. Once coated, rough cut trenches were milled around the bacteria to depths of $250 \mathrm{~nm}$ using an accelerating voltage of $5 \mathrm{kV}$, and current of $6.5 \mathrm{nA}$. Auto Slice and View software (FEI) was used to carry out sequential sectioning of S. aureus and E. coli in $30 \mathrm{~nm}$ slices. This was performed with an accelerating voltage of $5 \mathrm{kV}$, and beam current of $47.5 \mathrm{pA}$. Images of each section were acquired using electron beam accelerating voltages of $5 \mathrm{kV}$ and current of $98 \mathrm{pA}$. Secondary electron emission was detected using an Everhart-Thornley detector.

\section{TEM and FIB-SEM image processing and 3D volume reconstruction. TEM} cross sectional analysis was used to determine the frequency of nanopillar-induced envelope deformation and penetration in S. aureus, E. coli or K. pneumoniae. Frequencies are reported as percentages of the total cells analysed; 59 (S. aureus), 42 (E. coli) or 80 (K. pneumoniae). To construct tomograms of bacteria, raw tilt series data sets were processed using eTomo software. Tomograms were uploaded into Avizo v9.7.0 (FEI), and the segmentation editor was used to generate 3D volumes of each tomogram. The slice and view data acquired from sequential FIB milling was processed using the FIB-stack wizard tool in Avizo v9.7.0 (FEI). Briefly, this tool facilitates in aligning the FIB-stack and correcting geometrical artefacts, such as stage tilt foreshortening and/or vertical shift. Following data alignment and shearing correction within the FIB-stack wizard, image denoising was performed using a non-local means filter. Within Avizo segmentation editor, regions of bacteria and nanopillar were assigned as different materials, and were manually traced in consecutive orthoslices using the brush tool. The interpolation function was not used to join neighbouring orthoslices. The generate surface function was used in repeatable algorithm mode with smoothing deselected to produce $3 \mathrm{D}$ volumes of bacteria and nanopillars. 3D volumes were then simplified using the simplification editor in default mode. The smooth surface function was then applied to simplified 3D meshes of bacteria and nanopillars, with iteration and lambda functions set to $50-200$ and 0.6 , respectively.

Protein extraction from S. aureus and E. coli. Titanium discs $\left(25 \mathrm{~cm}^{2}\right)$ were placed inside sterile petri dishes $(94 \mathrm{~mm}$ by $16 \mathrm{~mm}$ ) and were inoculated with $1 \mathrm{~mL}$ S. aureus or E. coli suspensions containing $10^{7}-10^{8}$ cells. Titanium discs were incubated for $24 \mathrm{~h}$ at $37^{\circ} \mathrm{C}$ under static conditions inside a humidity chamber. Following incubation, titanium samples were rinsed in $20 \mathrm{~mL}$ Tris- $\mathrm{HCl}$ buffer to recover bacteria. Cells were harvested by centrifugation ( $5000 \mathrm{rpm}, 10 \mathrm{~min}$ ) and resuspended in Tris- $\mathrm{HCl}$. To extract S. aureus proteins, Tris- $\mathrm{HCl}(200 \mu \mathrm{L})$ was supplemented with $200 \mu \mathrm{g} / \mathrm{mL}$ lysostaphin (Sigma-Aldrich, St. Louis, MO, USA), and suspensions were incubated for $60 \mathrm{~min}$ at $37^{\circ} \mathrm{C}$ before cellular debris was removed by centrifugation at $13,300 \mathrm{rpm}$ for $20 \mathrm{~min}$ at $4{ }^{\circ} \mathrm{C}$. For E. coli proteins, cell suspensions $(500 \mu \mathrm{L})$ were snap frozen in liquid nitrogen and transferred to a pre-cooled $5 \mathrm{~mL}$ Teflon flask containing one tungsten carbide bead. Homogenisation of frozen bacteria was performed at $2000 \mathrm{rpm}$ for $2.5 \mathrm{~min}$, using a Micro-Dismembrator (Sartorius, Göttingen, Germany). This resultant powder was resuspended in $1 \mathrm{~mL}$ Tris- $\mathrm{HCl}(\mathrm{pH} 7.2)$ and cellular debris was removed by centrifugation at $13,300 \mathrm{rpm}$ for $20 \mathrm{~min}$ at $4^{\circ} \mathrm{C}$. Soluble S. aureus and E. coli protein fractions were then transferred to an Eppendorf tube and stored at $-80^{\circ} \mathrm{C}$.

TMT labelling and high pH reversed-phase chromatography. An equal volume of each protein sample was digested overnight at $37^{\circ} \mathrm{C}$ with $2.5 \mu \mathrm{g}$ trypsin, labelled with TMT ten plex reagents according to the manufacturer's protocol (Thermo 
Fisher Scientific), and the labelled samples pooled. An aliquot of the pooled sample was evaporated to dryness, resuspended in 5\% formic acid and then desalted using a SepPak cartridge according to the manufacturer's instructions (Waters, USA). Eluate from the SepPak cartridge was again evaporated to dryness and resuspended in buffer A (20 mM ammonium hydroxide, $\mathrm{pH} 10)$ prior to fractionation by high $\mathrm{pH}$ reversed-phase chromatography using an Ultimate 3000 liquid chromatography system (Thermo Scientific). In brief, the sample was loaded onto an XBridge BEH C18 Column (130 А, $3.5 \mu \mathrm{m}, 2.1 \mathrm{~mm} \times 150 \mathrm{~mm}$, Waters, UK) in buffer A and peptides eluted with an increasing gradient of buffer B $(20 \mathrm{mM}$ ammonium hydroxide in acetonitrile, $\mathrm{pH} 10$ ) from $0-95 \%$ over $60 \mathrm{~min}$. The resulting fractions were evaporated to dryness and resuspended in $1 \%$ formic acid prior to analysis by nano-LC MSMS using an Orbitrap Fusion Tribrid mass spectrometer (Thermo Scientific).

Nano-LC mass spectrometry. High $\mathrm{pH}$ reversed-phase fractions were further fractionated using an Ultimate 3000 nano-LC system in line with an Orbitrap Fusion Tribrid mass spectrometer (Thermo Scientific). In brief, peptides in 1\% (v/v) formic acid were injected onto an Acclaim PepMap C18 nano-trap column (Thermo Scientific). After washing with $0.5 \%(\mathrm{v} / \mathrm{v})$ acetonitrile/0.1\% (v/v) formic acid, peptides were resolved on a $250 \mathrm{~mm} \times 75 \mu \mathrm{m}$ Acclaim PepMap C18 reversephase analytical column (Thermo Scientific) over a 150 min organic gradient, using seven gradient segments (1-6\% solvent B over $1 \mathrm{~min}, 6-15 \%$ B over $58 \mathrm{~min}$, $15-32 \%$ B over $58 \mathrm{~min}, 32-40 \%$ B over $5 \mathrm{~min}, 40-90 \%$ B over $1 \mathrm{~min}$, held at $90 \%$ B for $6 \mathrm{~min}$ and then reduced to $1 \% \mathrm{~B}$ over $1 \mathrm{~min}$ ) with a flow rate of $300 \mathrm{nl} / \mathrm{min}$. Solvent A was $0.1 \%$ formic acid and Solvent B was aqueous $80 \%$ acetonitrile in $0.1 \%$ formic acid. Peptides were ionised by nano-electrospray ionisation at $2.0 \mathrm{kV}$ using a stainless steel emitter with an internal diameter of $30 \mu \mathrm{m}$ (Thermo Scientific) and a capillary temperature of $275^{\circ} \mathrm{C}$.

All spectra were acquired using an Orbitrap Fusion Tribrid mass spectrometer controlled by Xcalibur 2.0 software (Thermo Scientific) and operated in datadependent acquisition mode using an SPS-MS3 workflow. FTMS1 spectra were collected at a resolution of 120,000, with an automatic gain control (AGC) target of 200,000 and a max injection time of $50 \mathrm{~ms}$. Precursors were filtered with an intensity threshold of 5000, according to charge state (to include charge states 2-7) and with monoisotopic precursor selection. Previously interrogated precursors were excluded using a dynamic window $(60 \mathrm{~s}+/-10 \mathrm{ppm})$. The MS2 precursors were isolated with a quadrupole mass filter set to a width of $1.2 \mathrm{~m} / \mathrm{z}$. ITMS2 spectra were collected with an AGC target of 10,000, max injection time of $70 \mathrm{~ms}$ and CID collision energy of $35 \%$. For FTMS3 analysis, the Orbitrap was operated at 50,000 resolution with an AGC target of 50,000 and a max injection time of $105 \mathrm{~ms}$. Precursors were fragmented by high energy collision dissociation at a normalised collision energy of $60 \%$ to ensure maximal TMT reporter ion yield. Synchronous Precursor Selection (SPS) was enabled to include up to five MS2 fragment ions in the FTMS3 scan.

Proteomic data analysis. The raw data files were processed and quantified using Proteome Discoverer software v2.1 (Thermo Scientific) and searched against the UniProt S. aureus (strain Newman) database (downloaded October 2018; 2584 entries) or the Uniprot E. coli (strain K12) database (downloaded February 2019; 4469 entries), using the SEQUEST algorithm. Peptide precursor mass tolerance was set at $10 \mathrm{ppm}$, and MS/MS tolerance was set at $0.6 \mathrm{Da}$. Search criteria included oxidation of methionine $(+15.9949 \mathrm{Da})$ as a variable modification and carbamidomethylation of cysteine $(+57.0214 \mathrm{Da})$ and the addition of the TMT mass tag $(+229.163 \mathrm{Da})$ to peptide $\mathrm{N}$-termini and lysine as fixed modifications. Searches were performed with full tryptic digestion and a maximum of two missed cleavages were allowed. The reverse database search option was enabled, and all data were filtered to satisfy a false discovery rate of $5 \%$. Based on the protein abundance values from three biological replicates of each sample, a Student's $t$-test was used to determine the significance of protein abundance fold changes between $S$. aureus and E. coli incubated on NW-850-5 and control surface. Proteins with $P \leq 0.05$ were deemed significant. Blast2Go software was used to categorise S. aureus and $E$. coli DEPs based on GO terms; DEPs were grouped by Level 2 GO. Protein-protein interactions were investigated using the functional protein association network tool (STRING v11) within Cytoscape.

ROS-GIoTM $\mathbf{H}_{2} \mathrm{O}_{2}$ assay. As for the proteomic studies, S. aureus and E. coli were incubated on titanium discs (NW-850-5 or control) for $24 \mathrm{~h}$. After $18 \mathrm{~h}$, the $\mathrm{H}_{2} \mathrm{O}_{2}$ substrate solution was added to the discs, as per the manufacturer's protocol, before placing back into the incubator at $37^{\circ} \mathrm{C}$ for the remaining $6 \mathrm{~h}$. During this time, the $\mathrm{H}_{2} \mathrm{O}_{2}$ substrate reacts with $\mathrm{H}_{2} \mathrm{O}_{2}$, forming a luciferin precursor. After $24 \mathrm{~h}$, the ROS-Glo detection reagent was added. This converts the luciferin precursor into luciferin, which is then used by the Ultra-Glo ${ }^{\mathrm{TM}}$ Recombinant Luciferase to generate a luminescent signal that is proportional to the quantity of $\mathrm{H}_{2} \mathrm{O}_{2}$. Luminescence measurements were recorded using an automated Infinite ${ }^{\circledR} 200$ PRO microplate reader. Luminescence was recorded using automatic attenuation, $1 \mathrm{~s}$ integration time and $0.15 \mathrm{~s}$ settle time.

Statistical analysis. Statistical analyses were performed using IBM SPSS statistical package (version 25 ). For bacterial viability testing, statistically significant differences were determined using one-way analysis of variance (ANOVA), with a Tukey HSD post hoc test. For proteomic and oxidative stress response analyses, statistically significant differences were determined by Student's $t$-test. Statistically significant differences were attributed to variables with $P \leq 0.05$. Unless otherwise stated, values given are mean \pm standard deviation and are representative of three independent experimental replicates $(n=3)$, performed in triplicate.

Reporting summary. Further information on research design is available in the Nature Research Reporting Summary linked to this article.

\section{Data availability}

Source data underlying Figs. 8 and 10, and Supplementary Fig. 9, are available as a Source Data file. The mass spectrometry proteomics data have been deposited to the ProteomeXchange Consortium via the PRIDE partner repository with dataset identifier PXD017078. The UniProt S. aureus (strain Newman) database (downloaded October 2018; 2584 entries, Proteome ID: UP000006386) and the Uniprot E. coli (strain K12) database (downloaded February 2019; 4469 entries, Proteome ID: UP000000625) were used in this study. Other data supporting the findings of this study are available from the corresponding authors upon request.

Received: 29 October 2019; Accepted: 4 March 2020; Published online: 02 April 2020

\section{References}

1. Ivanova, E. P. et al. Natural bactericidal surfaces: mechanical rupture of Pseudomondas aeruginosa cells by cicada wings. Small 8, 2489-2494 (2012).

2. Ivanova, E. P. et al. Bactericidal activity of black silicon. Nat. Commun. 4, 1-7 (2013).

3. Kelleher, S. M. et al. Cicada wing surface topography: an investigation into the bactericidal properties of nanostructural features. ACS Appl. Mater. Interfaces 8, 14966-14974 (2016).

4. Bandara, C. D. et al. Bactericidal effects of natural nanotopography of dragonfly wing on Escherichia coli. ACS Appl. Mater. Interfaces 9, 6746-6760 (2017).

5. Nowlin, K., Boseman, A., Covell, A. \& LaJeunesse, D. Adhesion-dependent rupturing of Saccharomyces cerevisiae on biological antimicrobial nanostructured surfaces. J. R. Soc. Interface 12, 1-12 (2014).

6. Hasan, J. et al. Selective bactericidal activity of nanopatterned superhydrophobic cicada Psaltoda claripennis wing surfaces. Appl. Microbiol. Biotechnol. 97, 9257-9262 (2013).

7. Tripathy, A., Sen, P., Su, B. \& Briscoe, W. H. Natural and bioinspired nanostructured bactericidal surfaces. Adv. Colloid Interface Sci. 248, 85-104 (2017).

8. Lin, N., Berton, P., Moraes, C., Rogers, R. D. \& Tufenkji, N. Nanodarts, nanoblades, and nanospikes: mechano-bactericidal nanostructures and where to find them. Adv. Colloid Interface Sci. 252, 55-68 (2018)

9. Damiati, L. et al. Impact of surface topography and coating on osteogenesis and bacterial attachment on titanium implants. J. Tissue Eng. 9, 1-16 (2018)

10. Modaresifar, K., Azizian, S., Ganjian, M., Fratila-Apachitei, L. E. \& Zadpoor A. A. Bactericidal effects of nanopatterns: a systematic review. Acta Biomater. 83, 29-36 (2019).

11. Diu, T. et al. Cicada-inspired cell-instructive nanopatterned arrays. Sci. Rep. 4, 1-7 (2014).

12. Sjöström, T., Nobbs, A. H. \& Su, B. Bactericidal nanospike surfaces via thermal oxidation of Ti alloy substrates. Mater. Lett. 167, 22-26 (2016)

13. Hazell, G., Fisher, L. E., Murray, W. A., Nobbs, A. H. \& Su, B. Bioinspired bactericidal surfaces with polymer nanocone arrays. J. Colloid Interface Sci. 528, 389-399 (2018).

14. Pogodin, S. et al. Biophysical model of bacterial cell interactions with nanopatterned cicada wing surfaces. Biophys. J. 104, 835-840 (2013).

15. Xue, F., Liu, J., Guo, L., Zhang, L. \& Li, Q. Theoretical study on the bactericidal nature of nanopatterned surfaces. J. Theor. Biol. 385, 1-7 (2015)

16. $\mathrm{Li}, \mathrm{X}$. \& Chen, T. Enhancement and suppression effects of a nanopatterned surface on bacterial adhesion. Phys. Rev. E 93, 1-7 (2016).

17. Silhavy, T. J., Kahne, D. \& Walker, S. The bacterial cell envelope. Cold Spring Harb. Perspect. Biol. 2, 1-16 (2010).

18. Hazell, G. et al. Studies of black silicon and black diamond as materials for antibacterial surfaces. Biomater. Sci. 6, 1424-1432 (2018).

19. Rizzello, L. et al. Impact of nanoscale topography on genomics and proteomics of adherent bacteria. ACS Nano 5, 1865-1876 (2011).

20. Rizzello, L. et al. Molecular response of Escherichia coli adhering onto nanoscale topography. Nanoscale Res. Lett. 7, 2-8 (2012).

21. Kang, S., Herzberg, M., F. Rodrigues, D. \& Elimelech, M. Antibacterial effects of carbon nanotubes: size does matter! Langmuir 24, 6409-6413 (2008) 
22. Goriainov, V. et al. Harnessing nanotopography to enhance osseointegration of clinical orthopedic titanium implants-an in vitro and in vivo analysis. Front. Bioeng. Biotechnol. 6, 1-14 (2018).

23. Shah, F. A., Trobos, M., Thomsen, P. \& Palmquist, A. Commercially pure titanium (cp-Ti) versus titanium alloy (Ti6Al4V) materials as bone anchored implants - Is one truly better than the other? Mater. Sci. Eng. C 62, 960-966 (2016).

24. Lüdecke, C. et al. Nanorough titanium surfaces reduce adhesion of Escherichia coli and Staphylococcus aureus via nano adhesion points. Colloids Surf. B Biointerfaces 145, 617-625 (2016).

25. Dewald, C. et al. Gold nanoparticle contact point density controls microbial adhesion on gold surfaces. Colloids Surf. B Biointerfaces 163, 201-208 (2018).

26. Szklarczyk, D. et al. STRING v11: protein-protein association networks with increased coverage, supporting functional discovery in genome-wide experimental datasets. Nucleic Acids Res. 47, 607-613 (2019).

27. Shannon, P. et al. Cytoscape: a software environment for integrated models of biomolecular interaction networks. Genome Res. 13, 2498-2504 (2003).

28. Arts, I. S., Gennaris, A. \& Collet, J. F. Reducing systems protecting the bacterial cell envelope from oxidative damage. FEBS Lett. 589, 1559-1568 (2015).

29. Ezraty, B., Gennaris, A., Barras, F. \& Collet, J. F. Oxidative stress, protein damage and repair in bacteria. Nat. Rev. Microbiol. 15, 385-396 (2017).

30. Horsburgh, M. J., Clements, M. O., Crossley, H., Ingham, E. \& Foster, S. J. PerR controls oxidative stress resistance and iron storage proteins and is required for virulence in Staphylococcus aureus. Infect. Immun. 69, 3744-3754 (2001).

31. Fitzpatrick, T. B., Amrhein, N. \& Macheroux, P. Characterization of YqjM, an old yellow enzyme homolog from Bacillus subtilis involved in the oxidative stress response. J. Biol. Chem. 278, 19891-19897 (2003).

32. Silva, L. C. Nda et al. SOS response and Staphylococcus aureus: implication for drug development. Intech Chapter 6, 95-108 (2017).

33. Sugiyama, M. et al. Overproduction of the bleomycin-binding proteins from bleomycin-producing Streptomyces verticillus and a methicillin-resistant Staphylococcus aureus in Escherichia coli and their immunological characterisation. FEBS Lett. 362, 80-84 (1995).

34. Almarza, O., Núñez, D. \& Toledo, H. The DNA-binding protein HU has a regulatory role in the acid stress response mechanism in Helicobacter pylori. Helicobacter 20, 29-40 (2015).

35. Duran, E. C. \& Lucius, A. L. ATP hydrolysis inactivating Walker B mutation perturbs $E$. coli ClpA self-assembly energetics in the absence of nucleotide. Biophys. Chem. 242, 6-14 (2018).

36. Goemans, C. V., Vertommen, D., Agrebi, R. \& Collet, J. F. CnoX is a chaperedoxin: a holdase that protects its substrates from irreversible oxidation. Mol. Cell 70, 614-627 (2018).

37. Rahimpour, M. et al. GlgS, described previously as a glycogen synthesis control protein, negatively regulates motility and biofilm formation in Escherichia coli. Biochem. J. 452, 559-573 (2013).

38. Olivi, M. et al. Inhibition of microbial growth by carbon nanotube networks. Nanoscale 5, 9023-9029 (2013).

39. Hong, Y., Zeng, J., Wang, X., Drlica, K. \& Zhao, X. Post-stress bacterial cell death mediated by reactive oxygen species. Proc. Natl Acad. Sci. USA 116, 10064-10071 (2019).

40. Vadillo-Rodriguez, V., Schooling, S. R. \& Dutcher, J. R. In situ characterization of differences in the viscoelastic response of individual gram-negative and gram-positive bacterial cells. J. Bacteriol. 191, 5518-5525 (2009).

41. Shiu, C., Zhang, Z. \& Thomas, C. R. A novel technique for the study of bacterial cell mechanical properties. Biotechnol. Tech. 13, 707-713 (1999).

42. Dwyer, D. J. et al. Antibiotics induce redox-related physiological alterations as part of their lethality. Proc. Natl Acad. Sci. USA 111, e2100-e2109 (2014).

43. Kohanski, M. A., Dwyer, D. J., Hayete, B., Lawrence, C. A. \& Collins, J. J. A common mechanism of cellular death induced by bactericidal antibiotics. Cell 130, 797-810 (2007).

44. Zhao, X. \& Drlica, K. Reactive oxygen species and the bacterial response to lethal stress. Curr. Opin. Microbiol. 21, 1-6 (2014).

45. Vatansever, F. et al. Antimicrobial strategies centered around reactive oxygen species - bactericidal antibiotics, photodynamic therapy, and beyond. FEMS Microbiol. Rev. 37, 955-989 (2013).

46. Baker, J. et al. Copper stress induces a global stress response in Staphylococcus aureus and represses sae and agr expression and biofilm formations. Appl. Environ. Microbiol. 76, 150-160 (2010).
47. Mitchell, A. M. \& Silhavy, T. J. Envelope stress responses: balancing damage repair and toxicity. Nat. Rev. Microbiol. 17, 417-428 (2019).

48. Luttrell, T. et al. Why is anatase a better photocatalyst than rutile? - Model studies on epitaxial $\mathrm{TiO}_{2}$ films. Sci. Rep. 4, 1-8 (2014).

49. $\mathrm{Su}, \mathrm{R}$. et al. How the anatase-to-rutile ratio influences the photoreactivity of $\mathrm{TiO}_{2}$. J. Phys. Chem. C 115, 24287-24292 (2011).

50. Spurr, R. A. \& Myers, H. Quantitative analysis of anatase-rutile mixtures with an X-ray diffractometer. Anal. Chem. 29, 760-762 (1957).

51. James, E. H., Edwards, A. M. \& Wigneshweraraj, S. Transcriptional downregulation of agr expression in Staphylococcus aureus during growth in human serum can be overcome by constitutively active mutant forms of the sensor kinase AgrC. FEMS Microbiol. Lett. 349, 153-162 (2013).

52. Hautefort, I., Hautefort, I., Proenca, M. J., Jose, M. \& Hinton, J. C. D. Singlecopy green fluorescent protein gene fusions allow accurate measurement of. Society 69, 7480-7491 (2003).

53. Yu, C. L. et al. Application Booklet Leica EM CPD300 Automated Critical Point Dryer 1-51 (2014).

54. Benjamini, Y. \& Hochberg, Y. Controlling the false discovery rate: a practical and powerful approach to multiple testing. R. Stat. Soc. 57, 289-300 (1995).

\section{Acknowledgements}

We acknowledge funding from the Medical Research Council (MRC) Doctoral Training Programme (J.J.). BS and AHN would like to thank the MRC for funding (MR/N010345/ $1 \& \mathrm{MR} / \mathrm{S} 010343 / 1)$. We thank Wolfson Bio-imaging Facility and Proteomics Facility at the University of Bristol for their help with electron microscopy and quantitative proteomic analysis respectively. We thank the Henry Royce Institue for FIB-SEM access funding.

\section{Author contributions}

B.S. conceived the project and contributed to the editing of the manuscript. A.H.N. conceived the project and contributed to the editing of the manuscript. P.V. conceived the project. J.J. wrote the manuscript, performed all the experiments and data analysis, except for tilt series collection, cross section generation, and slice and view data collection, which was performed by J.M., C.N. and A.G., respectively.

\section{Competing interests}

The authors declare no competing interests.

\section{Additional information}

Supplementary information is available for this paper at https://doi.org/10.1038/s41467020-15471-x.

Correspondence and requests for materials should be addressed to A.H.N. or B.S.

Peer review information Nature Communications thanks Klaus Jandt, Enrica Pessione and the other, anonymous, reviewers for their contribution to the peer review of this work. Peer reviewer reports are available.

Reprints and permission information is available at http://www.nature.com/reprints

Publisher's note Springer Nature remains neutral with regard to jurisdictional claims in published maps and institutional affiliations.

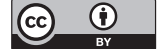

Open Access This article is licensed under a Creative Commons Attribution 4.0 International License, which permits use, sharing, adaptation, distribution and reproduction in any medium or format, as long as you give appropriate credit to the original author(s) and the source, provide a link to the Creative Commons license, and indicate if changes were made. The images or other third party material in this article are included in the article's Creative Commons license, unless indicated otherwise in a credit line to the material. If material is not included in the article's Creative Commons license and your intended use is not permitted by statutory regulation or exceeds the permitted use, you will need to obtain permission directly from the copyright holder. To view a copy of this license, visit http://creativecommons.org/ licenses/by/4.0/.

(C) The Author(s) 2020 